\title{
A Survey on Gait Recognition
}

\author{
CHANGSHENG WAN, Southeast University \\ LI WANG and VIR V. PHOHA, Syracuse University
}

Recognizing people by their gait has become more and more popular nowadays due to the following reasons. First, gait recognition can work well remotely. Second, gait recognition can be done from low-resolution videos and with simple instrumentation. Third, gait recognition can be done without the cooperation of individuals. Fourth, gait recognition can work well while other features such as faces and fingerprints are hidden. Finally, gait features are typically difficult to be impersonated.

Recent ubiquity of smartphones that capture gait patterns through accelerometers and gyroscope and advances in machine learning have opened new research directions and applications in gait recognition. A timely survey that addresses current advances is missing.

In this article, we survey research works in gait recognition. In addition to recognition based on video, we address new modalities, such as recognition based on floor sensors, radars, and accelerometers; new approaches that include machine learning methods; and examine challenges and vulnerabilities in this field. In addition, we propose a set of future research directions. Our review reveals the current state-of-art and can be helpful to both experts and newcomers of gait recognition. Moreover, it lists future works and publicly available databases in gait recognition for researchers.

CCS Concepts: • Computer systems organization $\rightarrow$ Artificial intelligence; Biometrics; Gait Recognition;

Additional Key Words and Phrases: Biometrics, gait recognition, individual identification

ACM Reference format:

Changsheng Wan, Li Wang, and Vir V. Phoha. 2018. A Survey on Gait Recognition. ACM Comput. Surv. 51, 5, Article 89 (August 2018), 35 pages.

https://doi.org/10.1145/3230633

\section{INTRODUCTION}

Biometric authentication, or simply biometrics [1-3], refers to identifying or verifying a person according to his/her biological or behavioral characteristics (also known as traits or identifiers). There are many biometric traits, such as fingerprints, face, and gait [4]. Typically, gait manifests a person's walking style. Gait recognition means authenticating a person by his/her manner of walking [5].

Early medical and psychological studies [6-8] showed that human gait had 24 different components, which could be used for identifying an individual. Moreover, in the previous works $[9,10]$,

The work is supported by NSFC Nos.: 61101088,71402070, NSF of Jiangsu Province No.: BK.20161099, and NSF No.: SaTC1527795.

Authors' addresses: C. Wan (corresponding author), L. Wang, and V. V. Phoha; emails: wan.changsheng@163.com, liwang32@yahoo.com, vir.phoha@gmail.com.

Permission to make digital or hard copies of all or part of this work for personal or classroom use is granted without fee provided that copies are not made or distributed for profit or commercial advantage and that copies bear this notice and the full citation on the first page. Copyrights for components of this work owned by others than ACM must be honored. Abstracting with credit is permitted. To copy otherwise, or republish, to post on servers or to redistribute to lists, requires prior specific permission and/or a fee. Request permissions from permissions@acm.org.

(C) 2018 Association for Computing Machinery.

0360-0300/2018/08-ART89 \$15.00

https://doi.org/10.1145/3230633 
it was shown that the light points attached to individuals' joints could be used for representing a human motion, and point light displays could be used for distinguishing human activities [10, 11]. All the above works indicated that every individual had unique muscular-skeletal structure, which could be used for identifying him/her [7]. Therefore, gait recognition is feasible.

Traditionally, gait-based authentication has been studied through analyzing the videos of human motion. However, the ubiquity of smart phones equipped with accelerometers and gyroscopes has opened a new dimension to gait-based authentication because of the unique ability to capture gait patterns by accelerometer and gyroscope. In addition, wide spread applications and advances in machine learning have resulted in improved accuracies. This survey is an attempt to bring a comprehensive, most up-to-date research and analysis of advances and the state of practice in gait-based authentication.

\subsection{Unique Properties of Gait Recognition}

Gait recognition has several unique properties that other biometrics lack [10, 12].

First, human gait can be captured far away from the individual. In contrast, other biometric systems [13-18] require that the individual is close to the biometric data collector or physically contacts it. This favorable characteristic has vital significance, while other biometric features such as fingerprints cannot be captured [19].

Second, gait recognition can be done at low resolution. When acquired videos are low resolution, other biometric systems such as face recognition may not work well. In this case, it is desired to use gait recognition [20].

Third, gait recognition can be done with simple instrumentation [12]. A camera, an accelerometer in the smartphone, a floor sensor, or even a radar can be used for collecting human gait.

Fourth, gait recognition can be done without the cooperation of the person. In contrast, other biometrics such as fingerprints require the individual putting his/her finger on the sensor for data acquisition.

Fifth, gait features are hard to be impersonated. This is because gait recognition typically uses human silhouettes and activities. This property is very important to crime analysis as discussed in Ref. [10].

Sixth, gait recognition still works well while features such as face images are hidden. It is easy to hide an individual's face, but hard to conceal human motions [10].

\subsection{Motivation}

Since gait recognition has so many unique properties, it has attracted much attention in the past 20 years. Several surveys on gait recognition have been done [5, 21-23]. These survey papers are described in Table 1.

However, these papers have the following issues:

(1) Existing papers only surveyed tens of research articles, and many important papers were not included.

(2) Existing surveys were done nearly 10 years ago, and many recent papers were not surveyed.

(3) Most survey papers focused on camera-based gait recognition systems, and survey on other gait recognition systems was limited. Though Gafurov [5] mentioned gait recognition systems using floor sensors and accelerometers, he neglected the introduction of concepts of various gait recognition systems. Nickel [24] reviewed around 35 accelerometerbased gait recognition systems in her doctoral thesis in 2012. She introduced sensors and datasets used in these systems and listed the equal error rates. However, the analysis of various techniques used in these accelerometer-based systems was not presented. 
Table 1. Lists of Existing Survey Papers on Gait Recognition

\begin{tabular}{|l|l|l|l|l|}
\hline Work & Reference [21] & Reference [22] & Reference [5] & Reference [23] \\
\hline NO & 60 & 33 & 33 & 30 \\
\hline Year & 2010 & 2011 & 2007 & 2009 \\
\hline DA & $\begin{array}{l}\text { 2D/3D } \\
\text { video-cameras }\end{array}$ & $\begin{array}{l}\text { 2D/3D } \\
\text { video-cameras }\end{array}$ & $\begin{array}{l}\text { 2D/3D video-cameras, } \\
\text { floor sensors, } \\
\text { accelerometers }\end{array}$ & $\begin{array}{l}\text { 2D/3D } \\
\text { video-cameras }\end{array}$ \\
\hline FR & $\begin{array}{l}\text { Model-based/ } \\
\text { Model-free }\end{array}$ & $\begin{array}{l}\text { Model-based/ } \\
\text { Model-free }\end{array}$ & $\times$ & $\begin{array}{l}\text { Model-based/ } \\
\text { model-free }\end{array}$ \\
\hline DR & LDA/PCA & $\times$ & $\times$ & PCA/LDA \\
\hline CL & SVM/DTW/HMM & $\times$ & $\times$ & $\times$ \\
\hline ND & 4 & $\times$ & $\times$ & $\times$ \\
\hline EF & $\times$ & $\times$ & $\times$ & $\times$ \\
\hline
\end{tabular}

Here NO means number of research articles surveyed, DA means Data Acquisition, FR means Feature representation, DR means Dimension Reduction, CL means Classification, ND means Number of datasets, EF means Environmental factors, and $\times$ means the techniques are not discussed by the survey paper.

(4) Existing survey papers only mentioned several model-based/model-free feature representation methods, and neglected the introduction of concepts of feature representation methods. More importantly, many features for accelerometer-based/floor-sensorbased/radar-based gait recognition systems were not mentioned.

(5) Existing survey papers only mentioned linear discriminant analysis (LDA) and principal component analysis (PCA) techniques for dimension reduction, and many other techniques such as outliers removals were neglected.

(6) Existing survey papers only mentioned a few classification techniques such as Support Vector Machine (SVM), Dynamic Time Wrapping (DTW), and Hidden Markov model (HMM), and many other classification techniques such as neural network classifiers were neglected.

(7) Existing survey papers only mentioned a few datasets, and many important publicly available datasets were neglected.

(8) Existing survey papers neglected the survey of environment factors, which would affect the gait recognition performance heavily. For example, as discussed in Section 9, environmental factors such as long time interval between two gait recognition attempts, clothing, walking speed, frame rate, and multi-view will reduce the recognition rate significantly.

To address the above issues in existing survey papers, we clarify terminologies and survey current research works in gait recognition systems.

This article is different from previous surveys in five ways.

(1) We include more than 100 gait recognition papers that were not discussed in the previous surveys.

(2) We present key techniques in gait recognition systems including data acquisition, feature representation, dimension reduction, and classification.

(3) We add references to publicly available datasets for gait recognition systems.

(4) We highlight environmental factors that affect gait recognition systems.

(5) And, we list the open research problems in gait recognition systems. 


\subsection{Organization}

The rest of this article is organized as follows. In Section 2, we introduce the framework and history of gait recognition systems. In Section 3, we present the data acquisition processes in gait recognition systems and introduce sensors used for collecting gait data. In Section 4, we survey feature representation methods in gait recognition systems. In Section 5, we analyze dimension reduction mechanisms in gait recognition systems. In Section 6, we present classification algorithms in gait recognition. In Section 7, we discuss publicly available datasets of gait recognition systems, which can be used for evaluating newly designed gait recognition systems. In Section 8, we discuss vulnerabilities of gait-based biometrics. In Section 9, we show how environmental factors affect the accuracy of gait recognition systems. In Section 10, we discuss future works and highlight open issues in gait recognition. Finally, in Section 11, we draw our conclusions.

\section{HISTORY AND FRAMEWORK OF GAIT RECOGNITION}

\subsection{History of Gait Biometrics}

In 1994, the earliest gait recognition system was proposed by authors in Reference [25], which was based on a small gait database. Then, the Defense Advanced Research Projects Agency (DARPA) [26] developed the famous HumanID program, which set up the first publicly accessible database for gait recognition. Since then, researchers have done many works on gait recognition.

Early gait recognition systems are mainly based on video. There are two sorts of video-based approaches, namely model-free and model-based approaches, as illustrated below.

Model-based approaches model human body and extract features from the model. In 1997, Cunado et al. [27] used the pendulum for modeling leg movement. And the transformation of legs' inclination in the video was used for gait recognition. In 2002, BenAbdelkader et al. [28] used cadence and stride for gait recognition. In 2003, Yoo et al. [29] modeled the human body as 2D figures, and computed trajectory-based kinematic features in the video for gait recognition. In 2004, Urtasun and Fua [30] introduced the 3D temporal models, while Wang et al. [31] took joint-angle trajectories of legs as features.

In contrast, model-free approaches did not model gait previously, and they represent the human gait as a whole without knowing the underlying structure of the human body. In 2006, Han and Bhanu [32] introduced the first model-free gait representation method named Gait Energy Image (GEI), which represented features in the video as a single image. In 2007, Liu and Zheng [33] introduced another gait representation method named Gait History Image (GHI). Then, they compared GHI with GEI and several other temporal templates.

Recently, gait recognition has been done through sensor readings such as accelerometer, floor sensors, and radars.

Accelerometer sensors are typically worn on human bodies. In 2005, Ailisto and Makela [34] introduced the first accelerometer-based gait system. Then, in Reference [35], Nakajima et al. used floor sensors for collecting gait data, such as pressure, for the first time. Finally, in 2005, Otero [36] first introduced the continuous wave radar for collecting gait data.

Table 2 presents the milestones of gait recognition systems. From Table 2, it can be seen that people have worked in the gait recognition area for around 20 years.

\subsection{Framework of Gait Biometrics}

Regardless of the underground techniques implemented, a typical gait recognition system (Figure 1) includes four phases, namely data acquisition, feature representation, dimension reduction, and classification. 
Table 2. Milestones of Gait Recognition

\begin{tabular}{|c|c|l|}
\hline Year & Work & \multicolumn{1}{c|}{ Description } \\
\hline 1994 & Reference [25] & First gait recognition system \\
\hline 1997 & Reference [27] & First model-based gait recognition system \\
\hline 2000 & Reference [35] & First floor-sensor-based gait recognition system \\
\hline 2005 & Reference [36] & First continuous-wave-radar-based gait recognition system \\
\hline 2005 & Reference [34] & First accelerometer-based gait recognition system \\
\hline 2006 & Reference [32] & First model-free gait recognition system \\
\hline
\end{tabular}

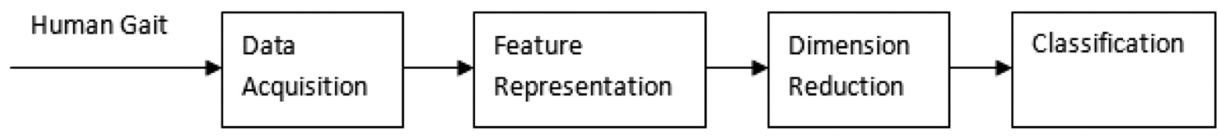

Fig. 1. Gait Recognition Overview.

Data acquisition. The data acquisition module is designed for collecting human gait data. Generally, human gait data is a set of temporal-spatial data, which includes activities of human body during a period of time. Specifically, human gait data can be collected in four ways, namely camera, accelerometer, floor sensor, and continuous wave radar. The features of human gait are included in the raw data collected during the data acquisition process.

Feature representation. To recognize individuals, the gait recognition system must extract gait features from raw data collected during the data acquisition phase. Feature representation is the first step for feature extraction, which converts raw data into specific styles. For videobased gait recognition, there are mainly two sorts of feature representation methods (modelbased/model-free methods). For example, Lee and Grimson [37] divide human silhouettes into seven parts and each part was fitted by an ellipse. Then, they took parameters of those ellipses as features. This is a typical model-based method. In Section 4, we further discuss feature representation methods.

Dimension reduction. In general, the dimensionality of features extracted from raw data in the feature representation step is higher than training data, resulting in the famous undersample problem. And the classification process will fail. Therefore, before classification, it is desired to extract useful features using a feature reduction algorithm. There are many dimension reduction methods, such as LDA and PCA [38]. In Section 5, we'll further discuss dimension reduction methods.

Classification. Direct gait classification methods are the simplest methods for gait recognition, which do not extract temporal features from gait sequences. Instead, this sort of method just uses several key frames in a gait sequence. Another approach is to use the similarity of gait temporal sequences for recognizing an individual. Recently, gait recognition systems have been built on HMMs [39] successfully.

\section{DATA ACQUISITION}

Gait raw data can be acquired in four ways, namely camera, accelerometer, floor sensor, and continuous wave radar. These four kinds of gait recognition systems are described below.

Camera. When people walk, their walking styles included in gait images are different, which can be used for identifying individuals. Typically, a camera is placed several meters away from objects for capturing videos. And the gait raw data is included in videos, which will be further processed for recognizing individuals. The Microsoft Kinect [40] is a widely used low cost RGB 
camera. Specifically, Microsoft Kinect can provide 3D skeletal images of 1-2 people. According to the work in Reference [40], Microsoft Kinect can achieve the same resolution as a laser scanner. However, Microsoft Kinect can only work well within the range of 3 meters [41]. There are many gait recognition systems based on Microsoft Kinect, such as References [40-43]. In general, there are two sorts of video-based gait approaches (model-based/model-free approaches) [8]. The modelbased approach aims to model individuals or activities and compute gait features according to the model [37]. Typically, the human motion can be modeled from both spatio and temporal data. For example, the ellipse model [37] is a typical spatio model. In this model, gait silhouettes are divided into seven regions, and one ellipse is used for fitting each region. And the gait features are elliptic parameters. In general, most gait models [27, 29, 30, 44, 45] used trajectory-based kinematic features of human joints as the pendulum for recognition. In contrast, model-free gait systems treat the silhouette as a whole, and extract features for identifying persons [23].

Accelerometer. When people walk, their accelerations in the 3D space are different, which can be used for identifying individuals. Typically, accelerometer sensors are put on human bodies. In accelerometer-based gait recognition systems, three-dimensional (X, Y, and Z) accelerations of an individual are recorded by accelerometers and further used for identifying an individual. This approach utilizes various kinds of sensors (e.g., accelerometer, gyroscope, and force sensors).

There are many accelerometer-based gait recognition systems. In Reference [46], a portable microprocessor-based data acquisition device was developed for measuring the three-dimensional gait acceleration signals during human walking. The system consisted of a triaxial accelerometer, an Microcontroller Unit (MCU), a 32MBe RAM, and a data transfer module for data transfer. This device was worn to the user's waist, and acceleration signals were sampled at a rate of $250 \mathrm{~Hz}$. Hoang [47] constructed an accelerometer-based gait recognition system with the classification accuracy approximately $91.33 \%$. In Reference [48], gait data was collected using an accelerometer equipped on the lower leg, which included forward-backward, sideways, and vertical accelerations. Derawi and Bours [49] presented the results of gait recognition on commercially available mobile smartphones, where both data acquisition and real-time analysis were done on the phone. In Reference [50], the accelerometer was placed in the trouser pocket, which could achieve a recognition rate of $86.3 \%$. Gafurov et al. [51] designed a gait collection device with two accelerometers, which could collect gait data in three directions.

Floor sensor. When people walk, their foot pressures are different, which can be used for identifying individuals. In this sort of gait system, many sensors such as the OR6-7 Force Platforms [52], the sensor mat [53], the load cells [54], and the EMFi floor [55] are deployed in the floor for collecting gait raw data when people walk on it.

Nakajima et al. [35] normalized the input raw footprint in five steps. By doing so, the influence of footprint position and direction was reduced, and the recognition rate was increased from $30.45 \%$ to $85.00 \%$. Suutala and Roning [55] designed a classification algorithm for footsteps, called Distinction-Sensitive Learning Vector Quantization (DSLVQ). And they also combined the LVQ algorithm together with other classifiers to improve the recognition rate [56,57]. Middleton et al. [53] aimed to design a low-cost floor-sensor-based gait recognition system. Orr and Abowd [54] showed the influence of footwear could be omitted. And Jenkins and Ellis [52] aimed to extract body mass information from footsteps.

Radar. When people walk, the motions of human body components (e.g., the torso, arms, and legs) generate different Doppler signatures, which can be used for identifying individuals [36].

Observing this, the continuous wave $(\mathrm{CW})$ radar placed several meters away from objects has been used for gait recognition. $\mathrm{CW}$ is a low-cost, low-power X-band microwave motion sensing module that is widely used for automatic door openers and security light actuators. In CW, a dielectric resonant oscillator (DRO) is used for generating signals, and two different antennas are 
Table 3. Gait Recognition Systems with Different Data Acquisition Methods

\begin{tabular}{|c|c|c|c|c|c|c|c|}
\hline Work & Sensor & Location & Data & EER (\%) & CCR (\%) & NS & ID \\
\hline Reference [60] & Camera & Front/side & Video & $4.3-9.5$ & - & 1,030 & 103 \\
\hline Reference [61] & Camera & Front/side & Video & 9.9-13.6 & - & 2,000 & 20 \\
\hline Reference [28] & Camera & Front/side & Video & 11 & - & 136 & 17 \\
\hline Reference [62] & Camera & Front/side & Video & $3.8-9$ & - & 13,640 & 124 \\
\hline Reference [63] & Camera & Front/side & Video & $8-14$ & - & 240 & 20 \\
\hline Reference [31] & Camera & Front/side & Video & $8-10$ & - & 80 & 20 \\
\hline Reference [64] & Camera & Front/side & Video & $7-23$ & - & 1,079 & 115 \\
\hline Reference [35] & Floor sensor & Floor & Foot pressure & - & 85 & 110 & 11 \\
\hline Reference [55] & Floor sensor & Floor & Foot pressure & - & $65.8-70.2$ & 200 & 11 \\
\hline Reference [56] & Floor sensor & Floor & Foot pressure & - & 79.2-98.2 & 440 & 11 \\
\hline Reference [53] & Floor sensor & Floor & Foot pressure & - & 80 & 360 & 15 \\
\hline Reference [54] & Floor sensor & Floor & Foot pressure & - & 93 & 1,680 & 15 \\
\hline Reference [52] & Floor sensor & Floor & Foot pressure & - & 39 & 62 & 62 \\
\hline Reference [65] & Accelerometer & Shoe & Velocity & - & 97.4 & 193 & 10 \\
\hline Reference [66] & Accelerometer & Shoe & Velocity & - & 96.93 & \begin{tabular}{|l|}
516,516 \\
\end{tabular} & 9 \\
\hline Reference [34] & Accelerometer & Waist & Velocity & 6.4 & - & 1,296 & 36 \\
\hline Reference [67] & Accelerometer & Waist & Velocity & $7-19$ & - & 11,556 & 36 \\
\hline Reference [68] & Accelerometer & Waist & Velocity & 6.7 & - & 175 & 35 \\
\hline Reference [46] & Accelerometer & \begin{tabular}{|l|} 
Waist \\
\end{tabular} & \begin{tabular}{|l|} 
Velocity \\
\end{tabular} & 5.6 & - & 105 & 21 \\
\hline Reference [69] & Accelerometer & Hand & Velocity & 17.2 & - & 62 & 31 \\
\hline Reference [69] & Accelerometer & Hip pocket & Velocity & 14.1 & - & 62 & 31 \\
\hline Reference [69] & Accelerometer & Breast pocket & Velocity & 14.8 & - & 62 & 31 \\
\hline Reference [47] & Accelerometer & Trouser pocket & Velocity & - & 91.33 & 1,054 & 14 \\
\hline Reference [70] & Accelerometer & Trouser pocket & Velocity & 22.49 & - & 204 & 51 \\
\hline Reference [49] & Accelerometer & Trouser pocket & Velocity & - & 89.3 & 375 & 25 \\
\hline Reference [71] & Accelerometer & Belt & Velocity & 5.7 & - & 3,960 & 60 \\
\hline Reference [72] & Accelerometer & Ankle & Velocity & 1.6 & - & 480 & 30 \\
\hline Reference [73] & Accelerometer & Hip pocket & Velocity & 21.7 & - & 2,592 & 48 \\
\hline Reference [36] & Radar & Front/side & Frequency & - & 95 & 142 & 49 \\
\hline
\end{tabular}

Here, frequency means spectrogram of radar micro-Doppler signals generated by human motions, EER means equal error rate, CCR means correct classification rate, NS means number of samples, and ID means identities.

used for transmitting and receiving signals. The output of the $\mathrm{CW}$ is a tone that represents the Doppler frequency of the moving human [36].

In Reference [58], A Ultra Wideband (UWB) impulse Doppler radar was developed for gait recognition, which could even detect vibrations of respiration and heartbeat for stationary human targets buried in collapse for search and rescue operations. Tahmoush and Silvious [59], collected gait data from a long range and showed how to extract features from radar micro-Doppler signals.

Table 3 summarizes the properties of current gait recognition systems with different data acquisition methods. From Table 3, we can see that

(1) Sensors are placed in different points in the four kind of gait recognition systems mentioned above. For example, camera-based and radar-based gait systems place sensors several meters around objects, floor-sensor-based gait systems place sensors in the ground, and accelerometer-based gait recognition systems place sensors all over human bodies. This variation results in different use in real-world scenarios. For instance, if the object 
is moving all over the world, an accelerometer-based gait recognition system is desired. And, if the object wants to pass the door, all the four kinds of gait recognition systems can work well.

(2) Different sensors generate different types of data. For example, a camera outputs video, a floor sensor outputs foot pressure, an accelerometer outputs velocity, and a radar outputs frequency. This will result in different processes in the subsequent recognition phases.

(3) The equal error rates of different systems are typically less than $25 \%$, and the correct classification rates are typically more than $60 \%$. Note that these numbers are calculated by individual research groups and depend on their used datasets.

(4) The number of samples is small. This shows that it is desired to build large databases for gait recognition.

\section{FEATURE REPRESENTATION}

Feature representation is the first step after data acquisition. Different data acquisition methods result in different feature representation techniques, as illustrated below.

\subsection{Model-based Feature Representation for Video Data}

Model-based feature representation aims to model the human body, and features are extracted from this model. Model-based feature representation is independent of scale and view. But it depends on the video quality [21].

The work in Reference [28] is the first model-based feature representation approach. BenAbdelkader et al. [28] modeled the human body using structural stride parameters of individuals. In Reference [28], two parameters, namely the stride length in meters and cadence (steps per minute), are computed from the gait video for recognizing individuals.

Most model-based feature representation methods aim to model the whole human body. For example, Yoo et al. [74] modeled the human body using 2D stick figures obtained by connecting the nine body points extracted from the gait. Boulgouris et al. [75, 76] divided the human body into multiple parts, too, and they mainly studied the importance of each part contributing to the recognition rate.

After modeling the whole human body, model-based feature representation methods typically use distances or joint angles on human body for gait recognition. For example, Bobick and Johnson [77] modeled the human body using four distances, namely left-right-foot, head-pelvis, head-foot, and foot-pelvis distances. Wang et al. [31] divided the human body into 14 parts and used jointangle trajectories in each part for identifying individuals. They also combined Procrustes shape analysis for improving recognition rate. Tanawongsuwan and Bobick [78] modeled the human body using joint angles. They extracted joint angles from gait data and used the trajectories of joint angles for recognizing individuals.

At the same time, since human legs play an important role in gait recognition, some works focused on the model of legs. For example, Yam et al. [44] modeled the human legs, and used them for analyzing walking and running. In this work, the recognition rate of running was higher. Dockstader et al. [45] modeled human legs, too, where legs were represented as many thick lines joining at one point. And Cunado et al. [79] used Velocity Hough transform (VHT) [80] for extracting features from legs and motions.

Table 4 summarizes current model-based feature representation techniques. From Table 4, we can see that:

(1) Model-based features typically include distances and angles of some points on human bodies. 
Table 4. Summary of Model-based Feature Representation Methods

\begin{tabular}{|c|l|l|l|l|l|}
\hline Work & \multicolumn{1}{|c|}{ Feature } & EER (\%) & \multicolumn{1}{c|}{ CCR (\%) } & \multicolumn{1}{c|}{ NS } & \multicolumn{1}{c|}{ ID } \\
\hline Reference [28] & Stride length/cadence & $11 \%$ & $40 \%$ & 136 & 17 \\
\hline Reference [77] & $\begin{array}{l}\text { Four distances (head-foot, head-pelvis, } \\
\text { foot-pelvis, left-right-foot) }\end{array}$ & - & $97.5 \%$ & 120 & 20 \\
\hline Reference [74] & 2D stick figure & - & $80-90 \%$ & 630 & 90 \\
\hline Reference [44] & Lower-leg pendulum, thigh pendulum & - & $80-90 \%$ & 100 & 20 \\
\hline Reference [45] & 25 parameters in the 3D stick image & - & $84-96 \%$ & 18,000 & 2 \\
\hline Reference [79] & $\begin{array}{l}\text { A set of features extracted using } \\
\text { velocity Hough transform }\end{array}$ & - & $80-100 \%$ & 40 & 10 \\
\hline
\end{tabular}

Here, EER means equal error rate, CCR means correct classification rate, NS means number of samples, and ID means identities.

(2) The correct classification rate of model-based schemes is typically more than $80 \%$, and the equal error rate is around $10 \%$.

(3) The dataset used in Reference [45] is large, while others are small.

\subsection{Model-free Feature Representation for Video Data}

Model-free feature representation aims to process the whole motion or shape of human silhouettes. Model-free feature representation has two main pros. First, it is independent of video quality [21]. This enables it to be deployed far away from the human object. Second, its computation cost is lower than model-based feature representation. Therefore, model-free feature representation is more popular. However, model-free feature representation has one main con: it depends on viewpoints and scale [21]. For example, when viewing angle changes, the gait recognition rate will be reduced as discussed in Reference [81].

The following paragraphs illustrate main model-free feature representation methods nowadays.

4.2.1 Direct Silhouette. The first model-free feature representation algorithm was proposed in Reference [82], which directly took silhouettes as features. In this work, the silhouette was the area of pixels from an individual, and the feature representation was to convert the sequence of images in the video to silhouettes for recognition. Sarkar et al. [82] extracted the silhouette from the background as follows.

First, the definition of a bounding box. Each frame of the video was split into two parts: the part within a manually defined bounding box and the outside part.

Second, the generation of the background information. From pixels of the outside part, the authors computed the background statistics. There were two statistics used in Reference [82], namely the mean and the covariances.

Third, the determination of the silhouette area. For each pixel within the bounding box, the authors computed the Mahalanobis distance between this pixel and the background. Then, the authors determined whether these pixels were background or foreground, according to this distance.

Finally, the gait feature was represented as a silhouette sequence.

In general, the above method can generate good quality silhouettes. However, there are several problems leading to segmentation errors [82]: (1) shadows; (2) threshold for classifying background and foreground; (3) moving object in the background; (4) compression artifacts at the human silhouette edge. 
4.2.2 Motion-History Image and Motion-Energy Image. In 2002, Bobick and Davis [83] introduced the motion-history image (MHI) and motion-energy image (MEI) methods, representing the silhouette sequence as a two-dimensional pattern.

The basic concept of MEI is that a sequence of silhouettes extracted in Reference [82] sweeps out an area, which can be used for identifying an individual. The MEI is defined below:

Given the image sequence $I(a, b, t)$ and the silhouette sequence $D(a, b, t) \in I$, the binary MEI is defined as $E_{\tau}(a, b, t)=\cup_{i=0}^{\tau-1} D(a, b, t-i)$, in which $\tau$ means the duration of the human motion, $a$ and $b$, are the coordinates in the image, and $t$ is the index in the sequence.

The MHI is a two-dimensional image for representing the movement of silhouettes, too. It computes current pixel $H_{\tau}(a, b, t)$ intensity from the previous one and the current image $D(a, b, t)$ as follows:

$$
H_{\tau}(a, b, t)=\left\{\begin{array}{r}
\tau, D(a, b, t)=1 \\
\max \left(0, H_{\tau}(a, b, t-1)-1\right), \text { otherwise }
\end{array} .\right.
$$

From the above definition, we can see that MEI and MHI can represent the moving part of individuals well, but they cannot represent the static part of individuals.

4.2.3 Gait History Image and Gait Energy Image. GEI [84] converts a sequence of silhouettes into a two-dimensional image too. However, different from MEI and MHI, GEI uses means of the silhouette sequence to construct a two-dimensional grey-level image. GEI is defined as follows:

Given a sequence of silhouettes $D(a, b, t)$, GEI is computed as $G(a, b)=\frac{1}{N} \sum_{t=1}^{N} D(a, b, t)$, in which $N$ is the frame total, $t$ is the frame index in the video, and $a$ and $b$ are coordinates in frames. GEI can reflect both static and dynamic parts of the silhouette sequence.

GEI has the following properties:

First, each silhouette in the sequence is a space-normalized energy image.

Second, GEI converts a sequence of silhouettes into an accumulative energy image, which is time-normalized.

Third, a high-intensity pixel in GEI means that the individual's activity at this point occurs frequently.

GHI [33] is developed from GEI, which is defined as:

$$
H_{\tau}(a, b, t)=\left\{\begin{array}{r}
\tau, S(a, b)=1 \\
\sum_{t=1}^{\tau} D(a, b, t)(t-1), \text { otherwise }
\end{array},\right.
$$

where $S(a, b)=\cap_{t=1}^{\tau} D(a, b, t)$ represents the non-moving pixels.

From the above definition, we can see that GEI and GHI can represent not only the moving part of individuals but also the non-moving part of individuals. Therefore, GEI and GHI are widely used nowadays.

4.2.4 Frame Difference Energy Image. Frame Difference Energy Image (FDEI) [85] stands for a variation of GEI. FDEI aims to repair the incompleteness of frames in the sequence of silhouettes, using the average values of clusters. FDEI includes four steps as defined below.

Step (1) Clustering and computing GEI. The sequence of silhouettes is divided into a number of clusters (e.g., temporally adjacent clusters). Then, the GEI of the $l$ th cluster $\left(C_{l}\right)$ is computed as $G_{l}(a, b)=\frac{1}{N_{l}} \sum_{t \in C_{l}} B(a, b, t)$, in which $B(a, b, t)$ is the frame of silhouette, and $t$ is the frame index. 
Step (2) Denoising. The GEI of the $l$ th cluster $\left(C_{l}\right)$ is denoised as:

$$
D_{l}(a, b)=\left\{\begin{array}{r}
G_{l}(a, b), \text { if } G_{l}(a, b) \geq T \\
0, \text { otherwise }
\end{array},\right.
$$

where $T$ is the threshold. $D_{l}(x, y)$ is named DEI.

Step (3) Computing the difference between frames. The difference between frames is computed as:

$$
F(a, b, t)=\left\{\begin{array}{c}
0, \text { if } B(a, b, t) \geq B(a, b, t-1) \\
B(a, b, t-1)-B(a, b, t), \text { otherwise }
\end{array} .\right.
$$

Step (4) Constructing FDEI. FDEI is computed as $F D(a, b, t)=F(a, b, t)+D_{l}(a, b)$.

4.2.5 Active Energy Image. Active energy image (AEI) was first introduced in Reference [86]. It can be used for representing relations among frames of the gait cycles. The AEI is computed from a silhouette sequence in two steps.

Step (1) Computing the difference between two silhouettes. The difference is computed as:

$$
F(a, b, t)=\left\{\begin{array}{r}
B(a, b, t), t=1 \\
|B(a, b, t)-B(a, b, t-1)|, t>1
\end{array} .\right.
$$

Step (2) Computing AEI. AEI is computed as $A(a, b)=\frac{1}{M} \sum_{t=1}^{M} F(a, b, t)$, in which $M$ is the frame total.

Finally, there are several more model-free feature representation methods. We summarize all of them in Table 5.

\subsection{Feature Representation for Accelerometer Data}

In this section, we first introduce two main feature representation methods for accelerometer data, namely gait-cycle-based and frame-based methods. Then, we introduce the fusion of features when multiple accelerometers are deployed.

4.3.1 Gait-cycle-based Feature Representation for Accelerometer Data. Gait-cycle-based feature representation [50, 73, 98, 99] aims to extract gait cycles from inertial signals and then generate an average cycle template from these cycles for classification. Typically, these sort of feature representation methods [99] include three phases:

Phase (1) Pre-processing. This phase is to fix data errors caused by data-acquisition sensors. First, the frequency of sensors may not be stable, and the number of data points acquired within the same time interval may vary. In this case, additional data points need to be inserted into the original data. Second, there may be noise in the acquired data, which must be reduced. Third, data of three directions will be combined into one resultant vector. For example, Derawi et al. [71, 100] computed the resultant vector using the following Equation (6) and Equation (7) respectively:

$$
\begin{aligned}
& R_{i}=\sin ^{-1}\left(\frac{c}{\sqrt{\left(a^{2}+b^{2}+c^{2}\right)}}\right), \\
& R_{i}=\sqrt{\left(a^{2}+b^{2}+c^{2}\right)},
\end{aligned}
$$

in which $a, b$, and $c$ are the horizontal, vertical, and sideways accelerometer values at observation $i$.

Phase (2) Step Detection. In general, one gait cycle includes two steps. And each step is determined by searching minimum points in the pre-processed data. For example, Holien detected 
Table 5. Summary of Model-free Feature Representation Methods

\begin{tabular}{|l|l|l|}
\hline \multicolumn{1}{|c|}{ Work } & \multicolumn{1}{|c|}{ Method } & \multicolumn{1}{c|}{ Description } \\
\hline Reference [82] & Direct Silhouette & $\begin{array}{l}\text { Silhouette quality is good. It may suffer from seg- } \\
\text { mentation errors. }\end{array}$ \\
\hline Reference [83] & MEI/MHI & $\begin{array}{l}\text { Motion-Energy Image/Motion-History Image. } \\
\text { They can represent the moving part of individuals } \\
\text { but cannot represent the static part. }\end{array}$ \\
\hline $\begin{array}{l}\text { References } \\
\text { [33, 84] }\end{array}$ & GEI/GHI & $\begin{array}{l}\text { Gait Energy Image/Gait History Image. They can } \\
\text { represent both moving and static parts. }\end{array}$ \\
\hline Reference [85] & FDEI & $\begin{array}{l}\text { Frame Difference Energy Image. It is a variation } \\
\text { of GEI for repairing the incompleteness of frames. }\end{array}$ \\
\hline Reference [86] & AEI & $\begin{array}{l}\text { Active Energy Image. It can represent relations } \\
\text { among frames. }\end{array}$ \\
\hline $\begin{array}{l}\text { References [8, } \\
87]\end{array}$ & Distance-based features & $\begin{array}{l}\text { Leftmost-rightmost-pixel width of the silhouette } \\
\text { is the feature. }\end{array}$ \\
\hline $\begin{array}{l}\text { References } \\
\text { 88, 89] }\end{array}$ & Centroid-based features & $\begin{array}{l}\text { Features are represented as Pixel-centroid dis- } \\
\text { tances. }\end{array}$ \\
\hline Reference [63] & Procrustes mean shape & Procrustes mean shape of the silhouette sequence. \\
\hline Reference [90] & Radon Features & $\begin{array}{l}\text { Features are generated using Radon transforma- } \\
\text { tion. }\end{array}$ \\
\hline Reference [91] & GEV & Gait Energy Volume. It is the 3D version of GEI. \\
\hline Reference [92] & PEI & Pose Energy Image. It is the mean image of frames. \\
\hline Reference [93] & PDV & $\begin{array}{l}\text { Pose Depth Volume. It is a combination of GEV } \\
\text { and PEI. }\end{array}$ \\
\hline Reference [94] & Learned Features & $\begin{array}{l}\text { Features are learned using convolutional neural } \\
\text { networks. }\end{array}$ \\
\hline Reference [95] & Learned Features & $\begin{array}{l}\text { Fatures are generated from supervised learning. } \\
\text { silhouette. }\end{array}$ \\
\hline Reference [96] & Local Features & $\begin{array}{l}\text { Gait Flow Image. It is generated from a sequence } \\
\text { of silhouettes using the optical flow field. }\end{array}$ \\
\hline Reference [97] & GFI & \\
\hline
\end{tabular}

steps using four algorithms [99], namely estimation of cycle length, indication of minimum value, detection of starting location, and detection of the total step.

Phase (3) Creation of the average cycle template. Finally, Holien [99] computed the mean value of multiple gait cycles, and got the average cycle template for classification.

4.3.2 Frame-based Feature Representation for Accelerometer Data. Frame-based feature representation [101] aims to divide inertial signals into multiple frames. For each frame, a feature vector is extracted. Finally, the sequence of feature vectors provides information about the gait, which will be used for classification. This sort of feature representation includes two phases as illustrated below.

Phase (1) Extraction of frame feature vector. There are a variety of feature vectors that can be extracted from time and frequency domains. Tang and Phoha [102] summarized the categories of features widely used in current accelerometer-based gait recognition systems, as shown in Table 6 . And Kumar et al. [103] summarized features from accelerometer and gyroscope in a smart phone, as shown in Table 7. 
Table 6. General Accelerometer and Gyroscope Features

\begin{tabular}{|c|c|c|}
\hline No. & Feature & Feature description \\
\hline 1 & Mean & $\begin{array}{l}\text { The mean value of } \mathrm{x}, \mathrm{y}, \mathrm{z} \text {-axis and } \mathrm{m} \text { within a time window. It } \\
\text { constitutes } 4 \text { features. }\end{array}$ \\
\hline 2 & Standard deviation & $\begin{array}{l}\text { The standard deviation within } \mathrm{x}, \mathrm{y}, \mathrm{z} \text {-axis and } \mathrm{m} \text { within a time } \\
\text { window. It constitutes } 4 \text { features. }\end{array}$ \\
\hline 3 & Range & $\begin{array}{l}\text { The difference between maximum and minimum value within } \mathrm{x} \text {, } \\
\mathrm{y}, \mathrm{z} \text {-axis and } \mathrm{m} \text { within a time window. It constitutes } 4 \text { features. }\end{array}$ \\
\hline 4 & Energy & $\begin{array}{l}\text { The energy of } \mathrm{x}, \mathrm{y}, \mathrm{z} \text {-axis and } \mathrm{m} \text { within a time window. It consti- } \\
\text { tutes } 4 \text { features. }\end{array}$ \\
\hline 5 & Spectral entropy & $\begin{array}{l}\text { It describes the complexity of the signal and is directly propor- } \\
\text { tional to the peak of the signal power spectrum and similar to } \\
\text { Shannon's entropy, with the use of the power spectral density as } \\
\text { a probability density. It constitutes } 4 \text { features. }\end{array}$ \\
\hline 6 & Bandpower & $\begin{array}{l}\text { The bandpower of a signal is defined as the average power in the } \\
\text { given frequency range ( } 0 \text { to Fs/2). This feature depends on the } \\
\text { sampling frequency (Fs) of the signal. It constitutes } 4 \text { features. }\end{array}$ \\
\hline 7 & Median frequency & $\begin{array}{l}\text { A frequency that divides the power spectrum into two regions } \\
\text { with equal amplitude is known as the median frequency. This } \\
\text { feature depends on the sampling frequency (Fs) of the signal. It } \\
\text { constitutes } 4 \text { features. }\end{array}$ \\
\hline 8 & $\begin{array}{l}\text { Inter Quartile } \\
\text { Range (IQR) }\end{array}$ & $\begin{array}{l}\text { The difference between signal quarter } 3 \text { (Q3) and quarter } 1 \text { (Q1). } \\
\text { It constitutes } 4 \text { features. }\end{array}$ \\
\hline 9 & Histogram (16 bins) & $\begin{array}{l}\text { It partitions the signal values into bins and returns the count in } \\
\text { each bin, as well as the bin edges. It constitutes } 96 \text { features. }\end{array}$ \\
\hline 10 & DTW distance & $\begin{array}{l}\text { DTW distance is computed between pair of signals. It constitutes } \\
3 \text { features. }\end{array}$ \\
\hline 11 & Mutual information & $\begin{array}{l}\text { Mutual information is computed between pair of signals. It con- } \\
\text { stitutes } 3 \text { features. }\end{array}$ \\
\hline 12 & Correlation & $\begin{array}{l}\text { Pearson correlation coefficient is computed between a pair of sig- } \\
\text { nals. It constitutes } 3 \text { features. }\end{array}$ \\
\hline
\end{tabular}

Phase (2) Model of the sequence of frame feature vectors. After feature extraction, some approaches aim to further model the sequence of feature vectors for each user. This model will be used for recognizing individuals. For example, San-Segundo et al. [101] used the Gaussian Mixture Model-Universal Background Model (GMM-UBM) for modeling the sequence of vectors for each individual. And Fraccaro et al. [104] further computed seven parameters from these frame vectors for classification, namely cadence, and six other stride parameters (velocity, length, time, Coefficient of Variation (CV) of time, CV velocity, and CV length).

4.3.3 Fusion of Features from Multiple Accelerometers. In some gait systems, multiple accelerometers are attached simultaneously on human bodies to get multiple gait signals. For example, Gafurov et al. [51] attached two accelerometers on the human body. In this case, signals from multiple accelerometers need to be fused.

A simple way for fusion is to generate a resultant vector using Equation (7) as shown in Reference [51]. To fuse complex features from multiple accelerometers, Chen et al. [105] introduced a Factorial Hidden Markov Model (FHMM) based scheme. In this scheme, data from each accelerometer is first transformed into an independent feature sequence. At time $t$, the combined feature $F_{t}$ 
Table 7. Features from Accelerometer and Gyroscope in a Smart Phone

\begin{tabular}{|l|l|l|}
\hline No. & Feature & \multicolumn{1}{|c|}{ Feature description } \\
\hline 1 & NOZC & No. of zero crossings \\
\hline 2 & Mean & Statistical mean \\
\hline 3 & ENG & Energy of the signal \\
\hline 4 & ABS & Absolute sum \\
\hline 5 & RNG & RNG (max(W) - min(W)) \\
\hline 6 & NOP & No. of peaks \\
\hline 7 & BAP & Power of the signal \\
\hline 8 & SEF & Spectral edge frequency \\
\hline 9 & SPE & Spectral entropy \\
\hline 10 & API & Average peak interval \\
\hline 11 & APL & Average peak length \\
\hline 12 & MED & Statistical median \\
\hline
\end{tabular}

is defined as $F_{t}=F_{t}^{1}, \ldots, F_{t}^{M}$, where $F_{t}^{m}$ is the feature in the $m$ th sequence at time $t$, and $M$ is the number of sequences. And the observation vector at time $t\left(O_{t}\right)$ depends on $F_{t}$. Then, the transition probability is computed as $P\left(F_{t} \mid F_{t-1}\right)=\prod_{m=1}^{M} P\left(F_{t}^{m} \mid F_{t}^{m-1}\right)$. Finally, Chen et al. defined two methods for computing the observation probability. In the first method, $O_{t}$ 's probability is a Gaussian random variable whose mean and covariance are computed from $F_{t}$. And in the second method, $O_{t}$ 's probability is the product of distributions of each sequence.

\subsection{Feature Representation for Floor-sensor Data}

The simplest way for representing floor-sensor data is body mass [52]. For example, body mass of a criminal is usually recorded in the database. In this case, the body mass information can be used for identifying this criminal. However, since many individuals may have the same body mass, the recognition rate of this feature representation may be low. Therefore, body mass is a weak biometric. Typically, body mass can be easily computed from pressure of the floor sensor as $M=$ $k F$, in which $F$ represents the pressure collected by the floor sensor, $k$ represents the coefficient, and $M$ represents the body mass.

More features can be extracted from floor-sensor data (pressure), such as cadence and length of stride [53]. Middleton et al. [53] placed 1,536 sensors in an area of $3 \mathrm{~cm}^{2}$. Stride length and stride cadence are used in video-based gait recognition systems too. Therefore, some video-based gait recognition algorithms can be used in floor-sensor-based gait recognition systems. Typically, these features can be easily computed from the pressure of the floor sensor. For example, the stride length can be computed from the heel strikes as $L_{s}=\left(h_{i+1}-h_{i}\right) s$, where $L_{s}$ is the stride length, $h_{i+1}$ and $h_{i}$ are sensor indices, and $s$ is the sensor spacing.

At the same time, Orr and Abowd [54] extracted seven features from the footstep profile, namely four parameters in the profile (length, standard deviation, mean, and area), and three coordinates in the profile.

Finally, Suutala and Roning [55] extracted 31 features from a single footstep including features from both the spatial and the frequency domain. These features are listed in Table 8 , where $(x, y)$ is the coordinate on the floor and fft means fast Fourier transformation.

\subsection{Feature Representation for Radar Data}

When a receiver and a transmitter are moving, the received frequency $\left(F_{d}\right)$ is different from the transmitted one $\left(F_{t}\right)$. This is called the Doppler frequency shift. Given the subject-radar-beam 
Table 8. Floor-sensor-based Features in Reference [55]

\begin{tabular}{|c|c|c|}
\hline No. & Feature & Feature description \\
\hline 1 & $x_{\max 1}, y_{\max 1}$ & Heel strike (maximum time and amplitude) \\
\hline 2 & $x_{\text {heel }}$ & Heel strike (start point) \\
\hline 3 & $x_{\max 2}, y_{\max 2}$ & Ball strike (maximum time and amplitude) \\
\hline 4 & $x_{\text {ball }}$ & Ball strike (end point) \\
\hline 5 & $x_{\min }, y_{\min }$ & Foot strike (minimum time and amplitude, heel-ball) \\
\hline 6 & $x_{\text {end }}, y_{\text {end }}$ & End point and its amplitude (before film-resetting) \\
\hline 7 & $m_{1}, \sigma_{1}$ & Mean and standard deviation (beginning- $x_{\min }$ ) \\
\hline 8 & $m_{2}, \sigma_{2}$ & Mean and standard deviation $\left(x_{\min }-x_{m i d}\right)$ \\
\hline 9 & $m_{3}, \sigma_{3}$ & Mean and standard deviation $\left(x_{\text {end }}-x_{\text {mid }}\right)$ \\
\hline 10 & $m_{\max }$ & Mean $\left(y_{\max 1}-y_{\max 2}-y_{\min }\right)$ \\
\hline 11 & $a_{1}$ & Area (beginning- $x_{\min }$ ) \\
\hline 12 & $a_{2}$ & Area $\left(x_{\min }-x_{\text {mid }}\right)$ \\
\hline 13 & length heel & Length $\left(x_{\min }-x_{\text {heel }}\right)$ \\
\hline 14 & length $_{\text {ball }}$ & Length $\left(x_{\text {ball }}-x_{\min }\right)$ \\
\hline 15 & $s_{\text {ball }}, s_{\text {heel }}$ & $\left(y_{\min }-y_{\max 2}\right) /\left(x_{\text {mid }}-x_{\text {ball }}\right),\left(y_{\min }-y_{\max 1}\right) /\left(x_{\text {heel }}-x_{\min }\right)$ \\
\hline 16 & $x_{\text {rel1 }}$ & Relation $\left(x_{\max 1} / x_{e n d}\right)$ \\
\hline 17 & $x_{\text {rel2 }}$ & Relation $\left(x_{\max 2} / x_{\text {end }}\right)$ \\
\hline 18 & $y_{\text {rel1 }}$ & Relation $\left(y_{\max 1} / x_{\text {end }}\right)$ \\
\hline 19 & $y_{\text {rel2 }}$ & Relation $\left(y_{\max 2} / x_{\text {end }}\right)$ \\
\hline 20 & $\mathrm{fft} 1, \mathrm{fft} 2, \mathrm{fft} 3, \mathrm{fft} 4$ & Amplitude spectrums \\
\hline
\end{tabular}

elevation angle $(\phi)$, the motion-radar-beam angle $(\theta)$, the light speed $(c)$, and the object-moving speed $(v)$, the relation between $F_{d}$ and $F_{t}$ is shown in Equation (8):

$$
F_{d}=F_{t} \frac{2 v}{c} \cos (\theta) \cos (\phi) \text {. }
$$

Since different parts of the human body have different speed $v$ during walking, the radar data converted by the short time Fourier transform will construct a spectrogram. From the spectrogram, Tahmoush and Silvious [59] extracted the stride rate from radar signals for gait recognition in five steps. In addition to the stride rate, the authors also used the radar cross section (RCS) for gait recognition.

Moreover, Otero [36] pointed out that the Doppler signature was mainly comprised of the motion of the torso and the legs, while the influence of the arms was not so important.

Finally, Wang and Fathy [58] showed that the ultra-wide-band Doppler radar could even acquire the breathing and heartbeat signatures for stationary human objects.

\section{DIMENSION REDUCTION}

Dimension reduction methods are used for removing non-important or bad features. It can be categorized into two types, namely feature reduction and outliers removal, as illustrated below.

\subsection{Feature Reduction}

Feature Reduction is a kind of dimension reduction method that can remove some irrelevant features to boost the gait biometric system's effectiveness and efficiency. There are many feature reduction methods, as discussed below.

5.1.1 Piecewise Linear Representation (PLR). PLR [106, 107] is a simple approach for feature reduction. It can discard minor fluctuations and keep major parts of signals of time series on signals. 
For example, Lawson and Duric [106] used Piecewise Linear function for representing edges of silhouettes as follows: If an edge element is at position $r$ at time $t$, the position of that edge element at time $t+\Delta t$ will be represented as $r+\Delta t \delta r_{n}$, where $r_{n}$ is the orthogonal edge direction.

5.1.2 Principal Component Analysis (PCA). PCA [108-112] is the most popular feature reduction method nowadays. It seeks to represent all $n$ data vectors as linear combinations of a small number of eigenvectors, and filter out the sample points, which are deviating from the norm. The detailed description of PCA training on gait data can be found in Reference [108], where Cheng et al. selected $k$ biggest eigenvectors for constructing a PCA eigentransformation matrix $W_{P C A}$, and then used $W_{P C A}$ for projecting original gait data into $k$-dimensional eigenspace. For example, BenAbdelkader et al. [112] introduced a scheme based on self-similarity plots (SSP) and PCA. Zhang et al. [110] first used dynamic gait energy image (DGEI) for representing gait data, and then used PCA and Locality preserving projection (LPP) for discovering the low-dimensional manifold of the high feature space. Lu et al. [111] used tensors instead of vectors, and extended traditional PCA to multilinear PCA (MPCA). And Xu and Zhang [109] developed a fuzzy PCA technique, which could diminish the influence of outliers.

5.1.3 Linear Discriminant Analysis (LDA). As a supervised machine learning feature detection method, through training, LDA $[90,108,113]$ can find a vector space $h$, which can well differentiate the data after mapping $Y=h X$ between classes and let data after mapping within classes get closer. Feature reduction is realized by such mapping onto vector space. Typically, LDA is used together with PCA. For example, Cheng et al. $[108,113]$ first used PCA for representing silhouettes in a lowdimensional space, and then used LDA for increasing separability. However, LDA can also be used together with other transforming methods. For example, Boulgouris and Chi [90] first performed Radon transform on binary silhouettes, and then used LDA for subspace projection.

5.1.4 Discrete Cosine Transform (DCT). DCT $[114,115]$ is another feature reduction approach that uses cosine functions for expressing gait signals. By doing so, it can reduce the correlation between variables and remove the frequency components that only have little contribution to the energy. For example, Fan et al. [114] first represented gait silhouettes using GEI. Then, for a $N \times N$ GEI image, its 2D DCT was:

$$
F(u, v)=a(u) a(v) \sum_{s=0}^{N-1} \sum_{t=0}^{N-1} f(s, t) \cos \left(\frac{(2 s+1) u \pi}{2 N}\right) \cos \left(\frac{(2 t+1) v \pi}{2 N}\right),
$$

in which

$$
a(u), a(v)=\left\{\begin{array}{r}
\sqrt{1 / N}, u, v=1 \\
\sqrt{2 / N}, u, v=1,2, \ldots, N-1
\end{array}\right.
$$

and the pixel value at $(s, t)$ in GEI was $f(s, t)$. After DCT transformation, the frequency components that only have little contribution in $F(u, v)$ could be deleted. Finally, the LDA technique was performed to increase the separability.

5.1.5 I-vector. I-vector is an efficient feature reduction approach for gait recognition systems, which has been proposed by San-Segundo et al. [116] recently. Basically, the i-vector approach uses a GMM-UBM [101] for training a total variability matrix $T$, and then uses $T$ for computing an ivector for classification. In this approach, there are mainly four parameters, the GMM supervector $M$ and the UBM supervector $m$, both with dimension $d$, the $d \times e$ total variability matrix $T$, and the i-vector $w$ with dimension $e$, where $e$ is determined by validation subsets and $d$ is computed from number of mixtures and mean vector size in the GMM model. Since $M=m+T w$, the i-vector can 
be computed from $M, m$, and $T$. The experimental result by San-Segundo et al. showed that the equal error rate was $6.1 \%$.

5.1.6 Other Feature Reduction Methods for Gait Recognition. There are several more feature reduction methods. For example, to solve the undersample problem (USP), Tao et al. [117] proposed a gait recognition system using Gabor features and general tensor discriminant analysis. Rani and Arumugam [118] extracted and trained gait features using the Modified Independent Component Analysis (MICA), which could reduce the dimension of features. Ye and Wen [119] employed discrete wavelet transformation (DWT) for reducing data dimensionality and filtering the noise in the procedure of template extracting. Mowbray and Nixon [120] used Fourier Descriptors for reducing data dimensionality. Huang et al. [121] used canonical analysis for reducing data dimensionality. Kale et al. [8] used the frame-exemplar distance for reducing data dimensionality. Bouchrika and Nixon [122] used factor analysis for reducing data dimensionality. Lai et al. [123] discussed several feature reduction methods, such as sparse bilinear discriminant analysis (SBDA), general tensor discriminant analysis (GTDA), and discriminant analysis with tensor representation (DATER).

\subsection{Outliers Removal}

Outlier removal is an integral part of almost all machine learning training algorithms. Unlike feature reduction, which is deleting some non-important features, outliers removal focuses on removing "bad quality" signals. Outlier removal is important because it will affect the performance of PCA significantly, as illustrated in Reference [124]. There are mainly four sorts of outliers.

5.2.1 Outliers in One Frame. Some gait systems only use several regions of human bodies for recognition, and other regions can be treated as outliers. For example, Mashagba [125] used GMM [101] for partitioning each frame in the gait video into ankle, knee, hip, and outlier regions.

5.2.2 Outliers in Datasets. Outliers in datasets refer to abnormal gait sequences. For example, Vera et al. [126] established six different criteria to find exactly which stride waveforms in the dataset could be identified as an outlier.

5.2.3 Outliers in One Gait Video. Some gait cycles in one gait video, such as the start-up strides in the video, may be quite different from other strides, and can be treated as outliers. For example, $\mathrm{Wu}$ and Krishnan [127] treated gait cycles that were twice standard deviations greater or less than the median value as outliers.

5.2.4 Outliers Caused by Clothes. When people wear different clothes, part of their silhouettes may be quite different from their normal size, and can be treated as outliers. For example, Ghebleh and Moghaddam [128] studied the GEIs of the same person with different clothes. The results showed that the distance values in rows affected by clothes were higher than the distance values in other rows, and could be treated as outliers. So, the authors used thresholds to detect and eliminate these outliers.

Finally, the typical dimension reduction methods are listed in Table 9.

\section{CLASSIFICATION}

Classification is the final phase in gait recognition systems. We briefly describe the following core methods and the reported research works that use these methods: Distance, Correlation, Machine Learning, HMM, and Bayesian Classification. 
Table 9. Dimension Reduction Methods

\begin{tabular}{|c|l|l|l|}
\hline Work & \multicolumn{1}{c|}{ Type } & \multicolumn{1}{c|}{ Method } & \multicolumn{1}{c|}{ CCR } \\
\hline Reference [106] & Feature Reduction & PLR & $69-73 \%$ \\
\hline Reference [113] & Feature Reduction & LDA & $54-58 \%$ \\
\hline Reference [109] & Feature Reduction & PCA & $89.7 \%$ \\
\hline Reference [114] & Feature Reduction & DCT & $86.6-94.4 \%$ \\
\hline Reference [129] & Feature Reduction & SREFS & $76.9-99 \%$ \\
\hline Reference [127] & Outliers Removal & Standard deviations & $70-90 \%$ \\
\hline Reference [128] & Outliers Removal & Distance & $80-100 \%$ \\
\hline
\end{tabular}

Here, CCR means correct classification rate.

\subsection{Distance}

The most common and straightforward classification method is Distance, where the distances from collected gait data to the patterns in the database are calculated, and the decision is made according to a threshold preset on the distance.

Euclidean distance is the most representative in gait biometric systems, which is computed using Equation (11):

$$
D(A, B)=\sqrt{\sum_{i=1}^{n}\left(x_{i}-y_{i}\right)^{2}},
$$

in which $D(A, B)$ means Euclidean distance from testing data $A=\left(x_{1}, \ldots, x_{n}\right)$ to the center of each training class $B=\left(y_{1}, \ldots, y_{n}\right)$. Such Square Norm ED can be combined with many other classification methods on the final decision step. There are many schemes based on Euclidean distance. For example, Nickel et al. [130] used Euclidean Distance for classification, and the false match rate was around $4 \%-5 \%$.

Manhattan distance is the second type of distance used in gait recognition, which is calculated using Equation (12):

$$
D(A, B)=\sum_{i=1}^{n}\left|x_{i}-y_{i}\right|,
$$

in which $D(A, B)$ means Manhattan distance from testing data $A=\left(x_{1}, \ldots, x_{n}\right)$ to the center of each training class $B=\left(y_{1}, \ldots, y_{n}\right)$. In Reference [50], the authors used Manhattan distance to reduce computation costs.

DTW distance $[70,131,132]$ is the third type of distance used for computing the similarity between collected gait data and the patterns in the database. This method can be used in gait biometric, especially when the speed and time of these sequences are different. The DTW process is described as follows.

Given two sequences of gait data $Q=q_{1}, \ldots, q_{n}$ and $C=c_{1}, \ldots, c_{m}$, DTW first constructs a $m \times n$ matrix $M$, whose element $M_{i, j}$ is the distance between $q_{i} \in Q$ and $c_{j} \in C$. Then, the similarity of $Q$ and $C$ is the cumulative distance from $M_{0,0}$ to $M_{m, n}$, where the cumulative distance at $M_{i, j}$ is computed as $D(i, j)=\min (D(i, j-1), D(i-1, j), D(i-1, j-1))+d\left(q_{i}, c_{j}\right)$.

Muaaz [70] used DTW in accelerometer-based gait systems, and the false match rate was around 1\%-3\%. Boulgouris [131] showed that DTW was suitable for video-based gait systems, too. And Barth et al. [132] proposed a Multi-dimensional Subsequence DTW (msDTW) scheme, and the recognition rate could even be $97 \%-98 \%$.

K-Nearest Neighbor (K-NN) $[130,133]$ is the fourth type of distance, where collected gait data is classified by neighbors' voting. This gait data will be assigned into the class which has most weight among its $k$-nearest class neighbors. This weight for assignment usually is a reciprocal of 
average distance between the object needing to be classified and its neighbors belonging to each class. Bajwa et al. [133] combined K-NN and other techniques together for gait recognition and achieved a recognition rate of $98.7 \%$. Nickel et al. [130] used K-NN in gait recognition and achieved a false match rate of $3.97 \%$.

Finally, there are several other distances in gait recognition systems. For example, Huang et al. [96] defined an image-to-class distance based on local feature representation and the average recognition rate was $79.17 \%$.

\subsection{Correlation}

Correlation is a statistical relationship involving dependence. Pearson's correlation coefficient [134-136], commonly called the correlation coefficient, is the most familiar measure of dependence. Given two sets of data $X=\left\{x_{1}, \ldots, x_{n}\right\}$ and $Y=\left\{y_{1}, \ldots, y_{n}\right\}$, the Pearson's correlation coefficient is computed in Equation (13), where $r_{X Y} \in[-1,1]$. If $\left|r_{X Y}\right|=1, X$ and $Y$ are completely correlated. In this situation, $X$ is linearly dependent on $Y$. The larger absolute value of $r$ signifies more similarity between $X$ and $Y$. When $\left|r_{X Y}\right|$ is larger then $0.8, X$ and $Y$ are in a high similarity status. When $\left|r_{X Y}\right|$ is smaller then $0.3, X$ and $Y$ are in low similarity status. Otherwise, $X$ and $Y$ are in medium similarity status.

$$
r_{X Y}=\frac{\sum_{i=1}^{n}\left(x_{i}-\bar{x}\right)\left(y_{i}-\bar{y}\right)}{\sqrt{\sum_{i=1}^{n}\left(x_{i}-\bar{x}\right)^{2} \sum_{i=1}^{n}\left(y_{i}-\bar{y}\right)^{2}}} .
$$

Since correlation coefficient can compute the similarity of two sets of data $X$ and $Y$, it is widely used in gait recognition systems. For example, Khurelbaatar et al. [136] used Pearson's correlation coefficient in their accelerometer-based gait systems, and the results showed that the correlation coefficient in the gait system was around 0.7-0.98. And Shmuel and Galit [134] summarized several Kinect-based gait systems that used correlation coefficient.

\subsection{Machine Learning}

Unlike outliers removal using an unsupervised machine learning method such as GMM, the machine learning classification for gait biometric systems is usually supervised. Through iteratively feeding gait features and corresponding labels into machine learning models in the training phase, machine learning classification can often capture some important information to represent subjects' identities, which the other classification cannot capture. The familiar machine learning classification for gait biometrics has SVM, LDA, Decision Tree Ensemble Classifier (DTEC), and neural network classifier. LDA can be implemented as both dimension reduction technique and as a classifier. Hence, we only introduce SVM, DTEC, and neural network classifier in this section.

6.3.1 SVM. SVM $[137,138]$ is a supervised machine learning classification method. When training, previously labeled gait data will be fed into SVM. Then, according to the SVM algorithm, the machine will find an appropriate line $(\vec{w} \cdot \vec{x}+b=0)$, which has a maximum distance to the lines $\vec{w} \cdot \vec{x}+b=1$ and $\vec{w} \cdot \vec{x}+b=-1$. When recognizing this, the line $\vec{w} \cdot \vec{x}+b=0$ can be used as a criterion for further classification. In the situation that the data cannot be linearly classified, a transform into a higher dimension space will be utilized. Wu and Wang [138] used PCA-based SVM to recognize young-elderly gait patterns and achieved a recognition rate of 90\%. Lu and Zhang [137] used independent component analysis and genetic fuzzy SVM for gait recognition, which even achieved a recognition rate of $100 \%$.

6.3.2 Decision Tree Ensemble Classifier. A decision tree is a predictor that utilizes a tree-like graph or model with many possible consequences to produce prediction according to the input. 
DTEC [139-141] is an aggregation of multiple weak decision trees $\left\{h\left(X, \Theta_{k}\right), k=1,2 \ldots K\right\}$, where $\Theta_{k}$ is the parameter set of the individual decision tree $k$, and $K$ represents the number of trees. The forming process of a DTEC and principle of feature importance estimation by DTEC are as follows:

(1) $K$ subsets are randomly extracted from the original training dataset via bagging. Corresponding to these $K$ subsets, $K$ decision trees are constructed and trained. For each extraction, the subset, which is not chosen, is named as "Out-of-Bag" $(\mathrm{OOB})$ data $\left(O O B_{k}\right)$;

(2) For each decision tree, if there are $n$ features, each time $m$ features are extracted $(m \leq n)$. The decision tree keeps choosing and splitting on the "most significant" feature $(f \in m)$ until it is fully grown;

(3) Aggregation at decision making is realized through the majority voting of these $K$ decision trees with weights $w_{k}\left(w_{k} \propto \frac{1}{E\left(O O B_{k}\right)}\right.$, in which $E$ is the error estimation function);

(4) The feature importance estimation is calculated by permuting one feature across OOB data and measuring how much worse the mean square error (MSE) of RFEC predictions becomes after the permutation.

Derlatka and Ihnatouski [139] used decision tree for gait recognition, and the recognition rate was $94 \%$. At the same time, the work in Reference [140] was based on the C4.5 decision tree algorithm.

6.3.3 Neural Network Classifier. Neural Network is computing inspired by neural structure of the brain, in which many neurones are highly interconnected for processing input data. This kind of Classification has been universally used in gait Biometric due to its ability to learn complicated relationships between the features extracted in the training phase.

Typically, a neural network contains three layers as described below. The input layer receives gait features. The hidden layer processes input features in a self-organizing manner. And the output layer outputs the class information of the input gait data.

There are many NN-based gait recognition systems. For example, Xiao and Yang [142] used Zernike Moments and Back Propagation (BP) Neural Network for gait recognition. And Su and Huang [143] used principal curves and neural networks for gait recognition.

6.3.4 Deep Learning in Gait Recognition. Building on the success of deep learning in fields such as image interpretation, computer vision, and natural language understanding, recently, deep learning methods have been successfully applied in gait recognition. Deep learning architectures, such as convolutional networks, deep neural networks, and deep belief networks, recurrent neural networks are characterized by multiple layers of neurons, and separate segments of the networks learn separate data representations. A key advantage of deep learning is that a separate feature creation is not necessary because the training mechanisms find the discriminative features from data. We briefly review the recent work in deep learning applications to gait recognition.

Alotaibi and Mahbub [144] develop a specialized architecture of deep convolutional neural networks for gait recognition. They use eight layers: four convolutional layers and four subsampling (pooling) layers. Their datasets consist of a 124 multi-view gait database, CASIA-B database [145]. They were able to achieve accuracies as high as $87.80 \%$. Yu et al. [146] used one deep model based on a multi-layer auto-encoder for invariant gait feature extraction and address variations imposed by view, clothing, and carrying conditions. They achieve state-of-art results on two large gait datasets (CASIA Gait Dataset B and SZU RGB-D). Sokolova and Konushin [147] implement deep learning for gait recognition using optical flow. They compare several deep neural network architectures. Castro et al. [148] use convolutional neural networks using optical flow to 
automatically learn gait features. Xing et al. [149] address cross view issues in gait recognition and propose a view-invariant gait recognition method by 3D convolutional neural network.

Gadaleta and Rossi [150] use deep learning on a smartphone-based gait authentication system. They use IDnet dataset with misclassification rates less than $0.15 \%$. Giorgi et al. [151] show very high accuracies on a dataset of 175 subjects wearing body inertial sensors. Dehzangi et al. [152] design a deep convolutional neural network to extract features from expanded gait cycles and jointly optimize the spatio-temporal features and identification model, although they had only 10 subjects and treated the identification problem as a 10-class classification problem. They achieved an accuracy of $97.06 \%$. Zhao and Zhou [153] encode a two-dimensional representation of gait from inertial data sequences in Angle Embedded Gait Dynamic Image (AE-GDI). AE-GDI is invariant to rotation and translation. They achieve comparable performance on McGill University dataset and Osaka University gait datasets.

\subsection{Hidden Markov Model}

HMM is widely used in gait recognition systems, and has many variations. Here, we only introduce several widely-cited HMM-based gait recognition systems.

Kale et al. [87] proposed a continuous HMM approach for gait recognition. In this approach, the authors trained the HMM using the width vectors derived from the sequences of gait silhouettes. And the HMM parameters $\lambda=(A, B, I I)$ were learned during training, in which $I I$ was the initial probability vector, and $B$ and $A$ were the observation and transition probability matrices, respectively. During the gait recognition phase, this approach first computed a sequence of probabilities from the HMM parameter and the probe data, and then compared it with instances stored in the database.

Kale et al. [8] estimated the observation probability and other HMM parameters using the imagefeature-exemplar distance. The results on several databases showed that the recognition rates were much higher than baselines.

In Reference [154], population HMM was used to normalize gait dynamics. And the biometric similarity between two normalized gait signatures was computed by summing the shape distances between the corresponding gait stances.

The GMM is a one state HMM, which can be used as a classification technique, too. For example, San-Segundo [101] used GMM for training frequency data from smart phones, and the total equal error rate is around $16.5 \%$.

The FHMM and the Parallel HMM (PHMM) are two variations for fusing multiple gait sequences [105]. FHMM is a feature-level fusion technique as shown in Section 4.3.3, and PHMM is a decision level fusion technique.

\subsection{Bayesian Classification}

The Bayesian rule can be used for computing the probability that a probe vector matches an instance in the database [64]. The Bayesian rule is described as $P(I \mid v)=\frac{P(v \mid I) P(I)}{P(v)}$, where $v$ is the probe vector and $I$ is the instance in database. Bazin [64] designed a Bayesian-classification-based gait system, and the equal error rate was around 5.9\%-15.5\%. And Manap et al. [155] used a Naive Bayes classifier for gait recognition.

\section{DATASETS}

To evaluate and compare the efficiency of newly designed gait recognition systems, publicly available datasets are developed. These datasets are described below. 


\subsection{Video-Based Datasets}

In 2001, Chalidabhongse et al. [156] published the UMD dataset. The UMD dataset is comprised of two walking-outside datasets. In the larger one, two cameras are placed orthogonally in a parking lot for collecting gait data, and there are 55 individuals walking $\mathrm{T}$ patterns. In addition, this dataset includes four views, namely back, left, right, and frontal.

In 2002, Phillips et al. [157] published the National Institute of Standards and Technology (NIST) Dataset, which was also well known as the HumanID dataset. The NIST dataset is comprised of 452 sequences, which are collected from 74 individuals. In front of two cameras, all these individuals walked around an ellipse. While walking, the following covariates were changed: flat concrete walking surface and grass lawn surface, sex, age, height, weight, foot dominance, shoes, heel height, and with or without briefcase.

In 2003, Wang et al. [88] published the CASIA dataset, in which dataset B was the most widely used. It included 124 individuals from 11 views and four walking styles with different speeds.

In 2004, Kale et al. [8] published the CMU dataset. The CMU dataset collected gait data from 25 individuals. In a 3D room, the authors placed six color cameras beside a treadmill for gait data acquisition, and individuals walked on this treadmill. There are four walking styles (incline/fast/slow walk, and with a ball).

In 2005, Sarkar et al. published the University of South Florida (USF) dataset [82], which included 1,870 gait sequences collected from 122 individuals. In front of cameras, all these individuals walked around an ellipse. While walking, the following five covariates were changed: with or without a briefcase, left viewpoint and right viewpoint, two different shoes types, two different time instants, and grass surface or concrete surface.

In 2007, Nixon and Carter published two gait datasets (large/small datasets) [158]. The small dataset collected gait data from 12 individuals. At a different speed, all individuals walked inside a track. While walking, the following three covariates were changed: without/with bags, clothes, and shoes. The large dataset collected more gait data than the small dataset. In the large dataset, the following covariates were changed while walking: six different views, inside/outside treadmill, and inside/outside track.

In 2014, Hofmann et al. published the TUM GAID datasets [159]. GAID is a multimodal gait dataset that includes gait data of audio, depth, and video. This gait data is collected from 305 individuals.

\subsection{Accelerometer-Based Datasets}

In 2005, Mantyjarvi et al. [67] published the Speed Dataset. This dataset collected gait data from 36 individuals. In this dataset, individuals were asked to walk with different speed (fast, normal, and slow). During data collecting, the accelerometer was placed on the individual's belt.

In 2007, Gafurov et al. [50] published the Motion-Recording-Sensor-Based Dataset. This dataset collected gait data from 50 individuals. In this dataset, individuals were asked to carry a $4 \mathrm{~kg}$ backpack or without a backpack, and to place the motion recording accelerometer in their right trouser pocket, during data collecting.

In 2012, Casale et al. [160] published the Walking Pattern Dataset. This dataset collected gait data from 22 individuals. In this dataset, walking patterns were sampled at $52 \mathrm{~Hz}$, and the accelerometer was embedded in an Andriod smartphone in the chest pocket. This dataset is publicly available.

In 2012, Muaaz and Nickel [161] published the Android phone Google G1 Dataset. This dataset collected gait data from 51 individuals. In this dataset, walking patterns of three-dimensional accelerometer data was first recorded at a $40-50 \mathrm{~Hz}$ sampling frequency. And then, this gait data was stored to a text file with timestamps on the SD-card. During data collecting, the phone was on the right side of the individual, attached using a belt. 
In 2014, Ngo et al. [162] published the largest accelerometer-based gait dataset. This dataset collected gait data from 744 individuals. In this dataset, there are half females and males, and the ages range from 2 to 78 years. Moreover, three accelerometers and one smartphone on different locations were used for collecting both angular and acceleration velocity data. This dataset is publicly available.

In 2016, Reyes et al. [163] published the Human Activities and Postural Transitions Dataset. This dataset collected gait data from 30 individuals. In this dataset, an embedded accelerometer and gyroscope were worn on the individual's waist. This dataset includes three dynamic motions (walking downstairs/upstairs and walking) and three static postures (standing, sitting, and lying). Moreover, this dataset includes six transitions between static postures (stand-to-sit, sit-to-stand, sit-to-lie, lie-to-sit, stand-to-lie, and lie-to-stand). This dataset is also publicly available.

In addition to the above datasets from healthy individuals, there exists a dataset from patients [164]. This dataset collected gait data from 10 Parkinson's disease patients and could be used for detecting freezing-of-gait events of patients.

\subsection{Floor-Sensor-Based Datasets}

In 2000, Orr and Abowd [54] published the first floor-sensor-based dataset. This dataset collected gait data from 15 individuals. In this dataset, individuals put on different shoes.

In 2004, Suutala and Roning [55] published a dataset that collected gait data from 11 individuals. In this dataset, about 200 footsteps of both feet were stored.

In 2005, Middleton et al. [53] published a dataset that collected gait data from 15 individuals. In this dataset, individuals were asked to walk without footwear.

In 2007, Jenkins and Ellis [52] published a dataset that collected gait data from 62 children. In this dataset, body mass was reported.

\subsection{Radar-Based Datasets}

In 2005, Otero [36] published the first wave-radar-based dataset. This dataset collected gait data from 49 individuals. In this dataset, individuals approached and receded from the radar.

In 2011, Wang and Fathy [58] published a dataset that collected gait data from one individual. In this dataset, the individual marched/walked with either one-arm or two-arm swings.

\section{VULNERABILITIES OF GAIT-BASED BIOMETRICS}

There are multiple similarities in vulnerabilities of gait and face biometrics. For example, both the face and the gait can be observed from a distance and without the knowledge of the individual. However, unlike face, gait possesses at least two unique characteristics: (1) it has a dynamic component composed of strides and cycles, and (2) clothing can obscure variations in gait patterns. Most work on spoofing (and anti-spoofing) the gait exploits these two characteristics.

Almost exclusively, spoofing attacks gait-based authentication studies have been confined to modalities that use video or that use sensors (in particular mobile phone based sensors) authentication. Even within the same modality (video or sensor-based authentication), different studies had different operating environments. For example, different studies had varying numbers of participants and different modes of attack, such as by matching the body type, by matching clothes worn, by trained actors who studied the walking patterns of subjects to be impersonated, and so on. We very briefly report these studies.

Spoofing attacks on Video-based gait authentication. Hadid et al. [165-167] showed successful impersonation of video-based gait biometrics. They suggested dividing the silhouette (and the body frame) into components and verifying each component separately. These studies exploited the clothes worn by the subject to launch attacks by using similar clothes by the 
impersonator as well as by choosing imposters with similar body characteristics. The chances of imposter attempts being successful are higher if the imposter and the victim are of the same gender.

Spoofing attacks on Sensor-based gait authentication. Mjaaland et al. [168] had a set of 50 participants in attacks on a sensor-based gait authentication system; they reported a natural boundary or plateau to the impostors performance; the value of this plateau predetermines the outcome of an attack. Muaz et al. [169] did two experiments. First, zero-effort attacks using a dataset of 35 participants were performed, and the EER was 13\%. Second, in impersonation attacks, five victims were paired with five attackers, and the attackers tried to mimic the walking style of the victims they were paired to. The results showed that it was difficult to mimic or walk like someone else. The studies by Gafurov [170] used 100 subjects. They concluded that minimal-effort impersonation attacks on gait biometric pose little threat; however, attackers with knowledge of their closest person can pose a serious threat to the authentication system. Kumar et al. [103] showed successful spoofs of gait-based biometrics through mobile phones. They matched feature values by adjusting the stride and pace with the help of a treadmill but required knowledge of the classifiers being used and the features that contributed most to the classification decisions, and thus, it was relatively difficult to launch.

In summary, the results in literature point to different levels of success in spoofing gait-based biometrics. Gait can be affected by the body type, clothes worn, and so on. Spoofing gait-based authentication is an active area of research and we expect significant advances in this field.

\section{ENVIRONMENTAL FACTORS THAT WILL AFFECT GAIT RECOGNITION}

There are many environmental factors that will affect gait recognition. These factors mainly include lengthy time interval, clothing, walking speed, frame rate, and multi-view, which are discussed below.

\subsection{Lengthy Time Interval}

Lengthy time interval can affect the gait recognition rate significantly. As discussed in Reference [171], after 6 months, the gait recognition rate could fall from $82 \%$ to $6 \%$. Liu et al. [172-174] also showed that lengthy time interval was non-negligible in gait recognition.

To address this issue, Veres et al. [171] proposed a predictive model of changes in gait. The assumptions in Reference [171] are listed below. First, the training dataset is available. Second, the relation of changes to subjects is fixed. The basic idea in Reference [171] is shown below. Given a gallery $G$, the predicted gallery $\hat{G}$ is computed as $\hat{G}=G+Q$, where $Q$ is a prediction matrix computed in four steps:

Step (1) The authors divided the gallery and the probe into $n$ groups, respectively.

Step (2) For each group $j \in[1, n]$, the authors computed the means of the gallery and the probe groups as $\bar{x}_{p}^{j}=\frac{1}{n^{j}} \sum_{i=1}^{n^{j}} x_{p i}^{j}$ and $\bar{x}_{g}^{j}=\frac{1}{n^{j}} \sum_{i=1}^{n^{j}} x_{g i}^{j}$, where $x_{p i}^{j}$ and $x_{g i}^{j}$ were records in the $j$ th gallery and probe group, respectively, and $n^{j}$ was the record total for the $j$ th group.

Step (3) For each group $j \in[1, n]$, the authors computed its prediction matrix as $Q^{j}=e\left(\bar{x}_{p}^{j}-\right.$ $\left.\bar{x}_{g}^{j}\right)$, where $e$ is a positive unit vector.

Step (4) The authors got the final prediction matrix as $Q=\left[Q^{1}, Q^{2}, \ldots, Q^{n}\right]$.

In 2012, Matovski et al. [175] proposed a gait recognition scheme based on Gait Energy Image (GEI) [84] and Gait Entropy Image (GEnI) [176]. This scheme can reduce the influence of lengthy time intervals and achieve a correct classification rate of $95 \%$. 
Finally, there are several publicly available datasets for lengthy time intervals. They are References $[26,37,87,177,178]$.

\subsection{Clothing}

Clothing may change gait features of individuals and reduce the recognition rate [179]. For example, a long coat worn on an individual will cover many important features.

To address this issue, Guan et al. [179] designed a scheme named random subspace method (RSM), which could achieve an average recognition accuracy of $80 \%$. In this work, Guan et al. used GEI [84] for gait feature representation and PCA [180] for preprocessing GEIs. Finally, the authors computed the similarity in the random subspaces for classification.

Finally, the well-known dataset that contains data sampled with different clothing syles is Reference [181].

\subsection{Walking Speed}

The walking speed may vary for the same individual. This variation will strongly reduce the recognition rate [182].

To address this issue, Kobayashi and Otsu [183] proposed a scheme based on the Fisher discriminant analysis. This scheme is comprised of three steps. First, the authors computed the high-order auto-correlation functions called the CHLAC features. Second, the authors mapped the CHLAC features to a feature vector using the Fisher discriminant analysis. Finally, K-NN was employed for recognition. Later, Aqmar et al. [182] combined the work in Reference [183] and HMMs [8] together to construct a training model.

Moreover, Lee et al. [184] proposed a shape-based frieze pattern, which could be used for representing relations between temporally separated gait motions, too.

Finally, the datasets with different walking speeds are the TokyoTech database [182], the University of Southampton (USH) large dataset [185], and the USF-NIST dataset A [186].

\subsection{Frame Rate}

Frame rate is another covariate factor that affects the gait recognition rate [187].

A typical way for solving this issue is to use the phase synchronization technique [188], which can match gait videos with different frame rates. However, when frame rates of gallery and probe are both low, phase synchronization may fail. To address this issue, Makihara et al. [189] increased the frame rate of gait sequence, and introduced a method named temporal Super Resolution. Another solution [187] is to compute an averaged single grayscale image from a sequence of gait images, and use this averaged image for gait recognition.

Finally, the well-known dataset that contains both high and low frame-rate data is the OU-ISIRD dataset [190].

\subsection{Multi-View}

Viewing angle variation will affect the gait recognition rate, too [81].

The simplest way for dealing with viewing angle variations is to compute the angle and transform the gait image using this angle. Early in 2000, Carter and Nixon [191] showed that the true thing angle $(\phi)$, the trajectory angle $(\theta)$, the measured angle $(\psi)$, and the inclined thing angle $(\alpha)$ had the following relation: $\tan (\phi)=\frac{\tan (\psi)}{\cos (\theta)}-\tan (\alpha) \tan (\theta)$.

Then, in 2002, Spencer and Carter [192] further included the camera elevation angle in the model of Ref. [191]. 
Later, in 2006, Makihara et al. [193] designed a view transformation model, which solved the viewing angle variation issue in the frequency domain. This model is based on Singular Value Decomposition.

All the above solutions aimed to transform viewing angles. Observing this, in 2011, Zheng et al. [81] developed a viewing invariant feature representation model using PCA.

\section{FUTURE WORK}

There are many works on gait recognition. However, few gait systems are deployed in real-world applications [21]. A large amount of work is desired as illustrated below.

First, further work for gender recognition is required. Gender recognition is a new kind of application that uses gait features. It has become more and more popular recently $[76,194]$ for the film industry, forensic implications, and medical implications. However, the recognition rate needs to be improved and covariates that affect the recognition rate need to be tested.

Second, further work for addressing the clothing covariate is required. Though the recognition rate of current schemes is relatively high, it may be affected by the clothing covariate. For example, if the individual holds a ball, or loads a package, or even wears a different pair of shoes, the recognition rate may fall down significantly [195]. So, more works are required to reduce the influence of the clothing covariate.

Third, further work for extracting gait silhouettes from complicated background is required. When an individual walks on a complicated surface, his/her silhouette is hard to be extracted by the gait recognition system. So, more work is required to reduce the influence of background [26].

Fourth, further work for addressing the multi-view covariate is required. When the same individual walks toward the camera with a different angle, the gait silhouettes extracted from the video data may be different. In this case, the gait recognition rate may fall down significantly. Current works mainly focused on the transforming model of different views. This may not be adequate, and more works such as training algorithms [196] are required to reduce the influence of multi-view.

Fifth, further work for combining gait and other biometrics such as face is required. Geng et al. [197] showed that the recognition rate could be improved by combining gait and face biometrics together. Therefore, more works are required to combine multiple biometrics together to achieve a high recognition rate.

Sixth, further work for constructing datasets is required. First, to improve the recognition rate of gait recognition systems, large datasets should be constructed. Second, datasets under different conditions such as complex background are required.

Seventh, further research on attacks on gait biometrics and counter measures to attacks is required. In general, it is hard to spoof gait recognition systems as shown in Reference [168]. However, under several exceptional circumstances, gait biometrics may be spoofed. For example, the results in [198] showed that if attackers knew their closest person in the dataset, they could establish efficient attacks. And Kumar et al. [103] showed that it would be very easy to spoof gait biometrics with the help of a treadmill, and the false acceptance rate could even be $70 \%$.

\section{CONCLUSION}

In this article, we have presented a detailed survey on gait recognition.

First, we have listed the history of gait recognition. Early gait recognition systems are built on video. In the past several years, the industry also built gait systems using floor sensors, accelerometers, and wave radars.

Second, we have discussed the framework of gait biometrics. It is mainly comprised of four modules, namely data acquisition, feature representation, dimension reduction, and classification. 
(1) Data acquisition. The gait data can be acquired by camera, accelerometer, floor sensor, and radar. Different acquisition method outputs different gait data.

(2) Feature representation. Different gait data can be represented in different ways. For videobased gait data acquired by camera, model-based and model-free feature representations are the main techniques. For gait data collected using accelerometer, floor sensor, and radar, there may be a variety of features, extracted in the frequency, time, and space domains.

(3) Dimension reduction. Typically, features extracted from gait data cannot be directly used for classification because the dimensionality of features extracted from raw data in the feature representation step is higher than training data. Therefore, a dimension reduction technique is needed. Dimension reduction can be divided into two types, namely feature reduction and outliers removal. Feature reduction aims to delete non-important features, while outliers removal aims to remove bad quality signals.

(4) Classification. Classification is the last phase of a gait recognition system, which identifies individuals. In this article, we mainly discussed seven kinds of classification algorithms, namely distance, correlation, machine learning, neural network, hidden Markov model, dynamic time wrapping, and Bayesian classification.

Third, we have analyzed the baselines of gait biometrics, which provided a benchmark for evaluating the performance of newly designed gait recognition systems. These baselines include publicly available datasets and corresponding algorithms.

Fourth, we have analyzed the vulnerabilities of gait-based biometrics and listed the environmental factors that were important to the performance. These environmental factors include lengthy time interval, clothing, walking speed, frame rate, and multi-view.

Finally, we have discussed the future works to be done.

\section{REFERENCES}

[1] Anil Jain, Ruud Bolle, and Sharath Pankanti. 2006. Biometrics: Personal Identification in Networked Society, Volume 479. Springer Science \& Business Media.

[2] Anil K. Jain, Arun Ross, and Salil Prabhakar. 2004. An introduction to biometric recognition. IEEE Transactions on Circuits and Systems for Video Technology 14, 1, 4-20.

[3] Ruud M. Bolle, Jonathan Connell, Sharath Pankanti, Nalini K. Ratha, and Andrew W. Senior. 2013. Guide to Biometrics. Springer Science \& Business Media.

[4] Anil K. Jain, Arun Ross, and Sharath Pankanti. 2006. Biometrics: a tool for information security. IEEE Transactions on Information Forensics and Security 1, 2, 125-143.

[5] Davrondzhon Gafurov. 2007. A survey of biometric gait recognition: Approaches, security and challenges. In Annual Norwegian Computer Science Conference, 19-21.

[6] M. P. Murray, A. B. Drought, and R. C. Kory. 1964. Walking patterns of normal men. fournal of Bone \& foint Surgery American Volume 46, 2, 335.

[7] M. P. Murray. 1967. Gait as a total pattern of movement: Including a bibliography on gait. American fournal of Physical Medicine \& Rehabilitation 46, 1, 290-333.

[8] Amit Kale, Aravind Sundaresan, A. N. Rajagopalan, Naresh P. Cuntoor, Amit K. Roy-Chowdhury, Volker Kruger, and Rama Chellappa. 2004. Identification of humans using gait. IEEE Transactions on Image Processing 13, 9, 1163-1173.

[9] Gunnar Johansson. 1973. Visual perception of biological motion and a model for its analysis. Perception \& Psychophysics 14, 2, 201-211.

[10] Mark S. Nixon and John N. Carter. 2004. Advances in automatic gait recognition. In Proceedings 6th IEEE International Conference on Automatic Face and Gesture Recognition, 2004. IEEE, 139-144.

[11] Geoffrey P. Bingham, Richard C. Schmidt, and Lawrence D. Rosenblum. 1995. Dynamics and the orientation of kinematic forms in visual event recognition. Journal of Experimental Psychology: Human Perception and Performance $21,6,1473$.

[12] Jeffrey E. Boyd and James J. Little. 2005. Biometric gait recognition. In Advanced Studies in Biometrics. Springer, $19-42$. 
[13] Anil K. Jain, Lin Hong, Sharath Pankanti, and Ruud Bolle. 1997. An identity-authentication system using fingerprints. Proceedings of the IEEE 85, 9, 1365-1388.

[14] Matthew A. Turk and Alex P. Pentland. 1991. Face recognition using eigenfaces. In Proceedings IEEE Computer Society Conference on Computer Vision and Pattern Recognition (CVPR'91). IEEE, 586-591.

[15] A. K. Jain and N. Duta. 1999. Deformable matching of hand shapes for verification. In Proceedings of International Conference on Image Processing. 857-861.

[16] John G. Daugman. 1993. High confidence visual recognition of persons by a test of statistical independence. IEEE Transactions on Pattern Analysis and Machine Intelligence 15, 11, 1148-1161.

[17] Lawrence R. Rabiner and Biing-Hwang Juang. 1993. Fundamentals of speech recognition. Prentice Hall.

[18] Yingyong Qi and Bobby R. Hunt. 1995. A multiresolution approach to computer verification of handwritten signatures. IEEE Transactions on Image Processing 4, 6, 870-874

[19] Nikolaos V. Boulgouris, Dimitrios Hatzinakos, and Konstantinos N. Plataniotis. 2005. Gait recognition: A challenging signal processing technology for biometric identification. IEEE Signal Processing Magazine 22, 6, 78-90.

[20] Daehee Kim and J. Paik. 2010. Gait recognition using active shape model and motion prediction. IET Computer Vision $4,1,25-36$

[21] Jin Wang, M. She, S. Nahavandi, and A. Kouzani. 2010. A review of vision-based gait recognition methods for human identification. In International Conference on Digital Image Computing: Techniques and Applications. $320-327$

[22] Zhaoxiang Zhang, Maodi Hu, and Yunhong Wang. 2011. A survey of advances in biometric gait recognition. In Chinese Conference on Biometric Recognition. Springer, 150-158.

[23] Ling Feng Liu, Wei Jia, and Yi Hai Zhu. 2009. Survey of gait recognition. In Intelligent Computing International Conference on Emerging Intelligent Computing Technology and Applications. 652-659.

[24] C. Nickel, Johannes Buchmann, and Christoph Busch. 2012. Accelerometer-based Biometric Gait Recognition for Authentication on Smartphones. PhD Thesis. Technische University, Darmstadt, Germany.

[25] Sourabh A. Niyogi, Edward H. Adelson, et al. 1994. Analyzing and recognizing walking figures in xyt. In CVPR, volume 94, pages 469-474.

[26] Sudeep Sarkar, P. Jonathon Phillips, Zongyi Liu, Isidro Robledo Vega, Patrick Grother, and Kevin W. Bowyer. 2005 The humanID gait challenge problem: Data sets, performance, and analysis. IEEE Transactions on Pattern Analysis and Machine Intelligence 27, 2, 162-177.

[27] David Cunado, Mark S. Nixon, and John N. Carter. 1997. Using gait as a biometric, via phase-weighted magnitude spectra. In International Conference on Audio-and Video-Based Biometric Person Authentication. Springer, 93-102.

[28] Chiraz BenAbdelkader, Ross Cutler, and Larry Davis. 2002. Stride and cadence as a biometric in automatic person identification and verification. In Proceedings of 5th IEEE International Conference on Automatic Face and Gesture Recognition, 2002. IEEE, 372-377.

[29] Jang-Hee Yoo and Mark S. Nixon. 2003. Markerless human gait analysis via image sequences. In Proceedings of International Society of Biomechanics 19th Congress. 1-5.

[30] Raquel Urtasun and Pascal Fua. 2004. 3D tracking for gait characterization and recognition. In Proceedings 6th IEEE International Conference on Automatic Face and Gesture Recognition, 2004. IEEE, 17-22.

[31] Liang Wang, Huazhong Ning, Tieniu Tan, and Weiming Hu. 2004. Fusion of static and dynamic body biometrics for gait recognition. IEEE Transactions on Circuits \& Systems for Video Technology 14, 2, 149-158.

[32] Ju Man and Bir Bhanu. 2006. Individual recognition using gait energy image. IEEE Transactions on Pattern Analysis and Machine Intelligence 28, 2, 316-322.

[33] Jianyi Liu and Nanning Zheng. 2007. Gait history image: A novel temporal template for gait recognition. In 2007 IEEE International Conference on Multimedia and Expo. IEEE, 663-666.

[34] Heikki J. Ailisto and Satu Marja Makela. 2005. Identifying people from gait pattern with accelerometers. Proceedings of SPIE-The International Society for Optical Engineering 5779, 7-14.

[35] K. Nakajima, Y. Mizukami, K. Tanaka, and T. Tamura. 2000. Footprint-based personal recognition. IEEE Transactions on Biomedical Engineering 47, 11, 1534.

[36] Michael Otero. 2005. Application of a continuous wave radar for human gait recognition. Proceedings of SPIE-The International Society for Optical Engineering 5809, 538-548.

[37] Lily Lee and W. Eric L. Grimson. 2002. Gait analysis for recognition and classification. In Proceedings 5th IEEE International Conference on Automatic Face and Gesture Recognition, 2002. IEEE, 155-162.

[38] Peter N. Belhumeur, João P. Hespanha, and David J. Kriegman. 1997. Eigenfaces vs. fisherfaces: Recognition using class specific linear projection. IEEE Transactions on Pattern Analysis and Machine Intelligence 19, 7, 711-720.

[39] Aravind Sundaresan, Amit RoyChowdhury, and Rama Chellappa. 2003. A hidden Markov model based framework for recognition of humans from gait sequences. In Proceedings 2003 International Conference on Image Processing, ICIP, Vol. 2. IEEE, II-93. 
[40] K. Khoshelham. 2012. Accuracy analysis of kinect depth data. ISPRS-International Archives of the Photogrammetry, Remote Sensing and Spatial Information Sciences 3812, 5, 133-138.

[41] A. Schmitz, M. Ye, R. Shapiro, R. Yang, and B. Noehren. 2014. Accuracy and repeatability of joint angles measured using a single camera markerless motion capture system. fournal of Biomechanics 47, 2, 587.

[42] B. Dikovski, G. Madjarov, and D. Gjorgjevikj. 2014. Evaluation of different feature sets for gait recognition using skeletal data from kinect. In International Convention on Information and Communication Technology, Electronics and Microelectronics. 1304-1308.

[43] Aaron Staranowicz, Garrett R. Brown, and Gian Luca Mariottini. 2013. Evaluating the accuracy of a mobile kinectbased gait-monitoring system for fall prediction. In Proceedings of the 6th International Conference on PErvasive Technologies Related to Assistive Environments. ACM, 1-4.

[44] ChewYean Yam, Mark S. Nixon, and John N. Carter. 2004. Automated person recognition by walking and running via model-based approaches. Pattern Recognition 37, 5, 1057-1072.

[45] Shiloh L. Dockstader, Michel J. Berg, and A. Murat Tekalp. 2003. Stochastic kinematic modeling and feature extraction for gait analysis. IEEE Transactions on Image Processing 12, 8, 962-976.

[46] Liu Rong, Jianzhong Zhou, Liu Ming, and Xiangfeng Hou. 2007. A wearable acceleration sensor system for gait recognition. In IEEE Conference on Industrial Electronics and Applications (ICIEA'07). 2654-2659.

[47] Thang Hoang. 2013. Adaptive cross-device gait recognition using a mobile accelerometer. fournal of Information Processing Systems 9, 2, 333-348.

[48] Davrondzhon Gafurov, Kirsi Helkala, and Torkjel Søndrol. 2006. Biometric gait authentication using accelerometer sensor. Fournal of Computers 1, 7, 51-59.

[49] Mohammad Derawi and Patrick Bours. 2013. Gait and activity recognition using commercial phones. Computers \& Security $39,39,137-144$.

[50] Davrondzhon Gafurov, Einar Snekkenes, and Patrick Bours. 2007. Gait authentication and identification using wearable accelerometer sensor. In 2007 IEEE Workshop on Automatic Identification Advanced Technologies. 220-225.

[51] D. Gafurov, K. Helkala, and T. Soendrol. 2006. Gait recognition using acceleration from mems. In International Conference on Availability. 432-439.

[52] Jam Jenkins and Carla Ellis. 2007. Using ground reaction forces from gait analysis: Body mass as a weak biometric. In Proceedings International ConferencePervasive Computing, Toronto, Canada, May 13-16, 2007. 251-267.

[53] Lee Middleton, Alex A. Buss, Alex Bazin, and Mark S. Nixon. 2005. A floor sensor system for gait recognition. In 4th IEEE Workshop on Automatic Identification Advanced Technologies, 2005. 171-176.

[54] Robert J. Orr and Gregory D. Abowd. 2000. The smart floor: A mechanism for natural user identification and tracking. In CHI 'O0 Extended Abstracts on Human Factors in Computing Systems. 275-276.

[55] Jaakko Suutala and Juha Röning. 2004. Towards the adaptive identification of walkers: Automated feature selection of footsteps using distinction-sensitive LVQ. Proceedings of the International Workshop on Processing Sensory Information for Proactive Systems (PSIPS'04).

[56] J. Suutala and J. Roning. 2005. Combining classifiers with different footstep feature sets and multiple samples for person identification. In Proceedings IEEE International Conference on Acoustics, Speech, and Signal Processing, 2005. Vol. 5, v/357-v/360

[57] Jaakko Suutala and Juha Roning. 2008. Methods for person identification on a pressure-sensitive floor: Experiments with multiple classifiers and reject option. Information Fusion 9, 1, 21-40.

[58] Yazhou Wang and Aly E. Fathy. 2011. Micro-doppler signatures for intelligent human gait recognition using a UWB impulse radar. In IEEE International Symposium on Antennas and Propagation. 2103-2106.

[59] Dave Tahmoush and Jerry Silvious. 2009. Radar micro-doppler for long range front-view gait recognition. In IEEE International Conference on Biometrics: Theory, Applications, and Systems. 1-6.

[60] Richard D. Seely, Sina Samangooei, Middleton Lee, John N. Carter, and Mark S. Nixon. 2008. The University of Southampton multi-biometric tunnel and introducing a novel 3D gait dataset. In IEEE International Conference on Biometrics: Theory, Applications and Systems. 1-6.

[61] Sungjun Hong, Heesung Lee, Kyongsae Oh, Mignon Park, and Euntail Kim. 2006. Gait recognition using sampled point vectors. In 2006 International foint Conference, SICE-ICASE. 3937-3940.

[62] Yuan Wang, Shiqi Yu, Yunhong Wang, and Tieniu Tan. 2006. Gait recognition based on fusion of multi-view gait sequences. In International Conference on Advances in Biometrics. 605-611.

[63] Liang Wang, Tieniu Tan, Weiming Hu, and Huazhong Ning. 2003. Automatic gait recognition based on statistical shape analysis. IEEE Transactions on Image Processing 12, 9, 1120-1131.

[64] Alex I. Bazin. 2005. Probabilistic combination of static and dynamic gait features for verification. Proceedings of SPIE-The International Society for Optical Engineering 5779, 4, 23-30.

[65] Morris and J. Stacy. 2004. A shoe-integrated sensor system for wireless gait analysis and real-time therapeutic feedback. PhD Thesis. Massachusetts Institute of Technology, Boston, USA. 
[66] Bufu Huang, Meng Chen, Panfeng Huang, and Yangsheng Xu. 2007. Gait modeling for human identification. In IEEE International Conference on Robotics and Automation. 4833-4838.

[67] J. Mantyjarvi, M. Lindholm, E. Vildjiounaite, and S. M. Makela. 2005. Identifying users of portable devices from gait pattern with accelerometers. In ICASSP. 973-976.

[68] L. Rong, D. Zhiguo, Z. Jianzhong, and L. Ming. 2007. Identification of individual walking patterns using gait acceleration. In The International Conference on Bioinformatics and Biomedical Engineering. 543-546.

[69] Elena Vildjiounaite, Mikko Lindholm, and Heikki Ailisto. 2006. Unobtrusive multimodal biometrics for ensuring privacy and information security with personal devices. In International Conference on Pervasive Computing. 187-201.

[70] Muhammad Muaaz. 2013. An analysis of different approaches to gait recognition using cell phone based accelerometers. In Proc. MOMM. 293-300.

[71] Mohammad O. Derawi, Patrick Bours, and Kjetil Holien. 2010. Improved cycle detection for accelerometer based gait authentication. In 6th International Conference on Intelligent Information Hiding and Multimedia Signal Processing. 312-317.

[72] D. Gafurov, E. Snekkenes, and P. Bours. 2010. Improved gait recognition performance using cycle matching. In IEEE International Conference on Advanced Information NETWORKING and Applications Workshops, WAINA 2010, Perth, Australia, April 13-20. 836-841.

[73] Claudia Nickel, Mohammad O. Derawi, Patrick Bours, and Christoph Busch. 2011. Scenario test of accelerometerbased biometric gait recognition. In International Workshop on Security \& Communication Networks. 15-21.

[74] Jang Hee Yoo, Doosung Hwang, Ki Young Moon, and Mark S. Nixon. 2008. Automated human recognition by gait using neural network. In 1st Workshops on Image Processing Theory, Tools and Applications, IPTA 2008. 1-6.

[75] Nikolaos V. Boulgouris and Zhiwei X. Chi. 2007. Human gait recognition based on matching of body components. Pattern Recognition 40, 6, 1763-1770.

[76] Xuelong Li, Stephen J. Maybank, Shuicheng Yan, Dacheng Tao, and Dong Xu. 2008. Gait components and their application to gender recognition. IEEE Transactions on Systems, Man, and Cybernetics, Part C (Applications and Reviews) 38, 2, 145-155.

[77] Aaron F. Bobick and Amos Y. Johnson. 2001. Gait recognition using static, activity-specific parameters. In Proceedings of the 2001 IEEE Computer Society Conference on Computer Vision and Pattern Recognition. CVPR 2001. Vol. 1. 423-430.

[78] R. Tanawongsuwan and A. Bobick. 2001. Gait recognition from time-normalized joint-angle trajectories in the walking plane. In IEEE Computer Society Conference on Computer Vision \& Pattern Recognition. 726.

[79] David Cunado, Mark S. Nixon, and John N. Carter. 2003. Automatic extraction and description of human gait models for recognition purposes. Computer Vision \& Image Understanding 90, 1, 1-41.

[80] Jason M. Nash, John N. Carter, and Mark S. Nixon. 1997. Dynamic feature extraction via the velocity Hough transform. Pattern Recognition Letters 18, 10, 1035-1047.

[81] Shuai Zheng, Junge Zhang, Kaiqi Huang, Ran He, and Tieniu Tan. 2011. Robust view transformation model for gait recognition. In IEEE International Conference on Image Processing. 2073-2076.

[82] S. Sarkar, P. J. Phillips, Z. Liu, I. R. Vega, P. Grother, and K. W. Bowyer. 2005. The humanID gait challenge problem: Data sets, performance, and analysis. IEEE Transactions on Pattern Analysis \& Machine Intelligence 27, 2, 162.

[83] Aaron F. Bobick and James W. Davis. 2002. The recognition of human movement using temporal templates. IEEE Transactions on Pattern Analysis \& Machine Intelligence 23, 3, 257-267.

[84] J. Han and B. Bhanu. 2006. Individual recognition using gait energy image. IEEE Transactions on Pattern Analysis \& Machine Intelligence 28, 2, 316-322.

[85] Changhong Chen, Jimin Liang, Heng Zhao, Haihong Hu, and Jie Tian. 2009. Frame difference energy image for gait recognition with incomplete silhouettes. Pattern Recognition Letters 30, 11, 977-984.

[86] Erhu Zhang, Yongwei Zhao, and Wei Xiong. 2010. Active energy image plus 2DLPP for gait recognition. Signal Processing 90, 7, 2295-2302.

[87] Amit A. Kale, Naresh Cuntoor, and Volker Krĺźger. 2002. Gait-based recognition of humans using continuous HMMs. In Proceedings IEEE International Conference on Automatic Face and Gesture Recognition, 2002. 336-341.

[88] Liang Wang, Tieniu Tan, Huazhong Ning, and Weiming Hu. 2003. Silhouette analysis-based gait recognition for human identification. IEEE Transactions on Pattern Analysis and Machine Intelligence 25, 12, 1505-1518.

[89] N. V. Boulgouris, K. N. Plataniotis, and D. Hatzinakos. 2004. An angular transform of gait sequences for gait assisted recognition. In International Conference on Image Processing, Vol. 2. 857-860.

[90] N. V. Boulgouris and Z. X. Chi. 2007. Gait recognition using radon transform and linear discriminant analysis. IEEE Transactions on Image Processing A Publication of the IEEE Signal Processing Society 16, 3, 731-740.

[91] Sabesan Sivapalan, Daniel Chen, Simon Denman, and Sridha Sridharan. 2011. Gait energy volumes and frontal gait recognition using depth images. In International foint Conference on Biometrics. 1-6. 
[92] Rotimi Ajayi. 2012. Gait recognition using pose kinematics and pose energy image. Signal Processing 92, 3 , 780-792.

[93] Pratik Chattopadhyay, Aditi Roy, Shamik Sural, and Jayanta Mukhopadhyay. 2014. Pose depth volume extraction from RGB-D streams for frontal gait recognition. Journal of Visual Communication \& Image Representation 25, 1 , $53-63$.

[94] Jia Ning, Sanchez Victor, and Li Chang-Tsun. 2017. Learning optimised representations for view-invariant gait recognition. In 2017 IEEE International foint Conference on Biometrics (IfCB). 774-780.

[95] Michal Balazia and Petr Sojka. 2017. You are how you walk: Uncooperative MOCAP gait identification for video surveillance with incomplete and noisy data. In IEEE International foint Conference on Biometrics. 208-215.

[96] Yi Huang, Dong Xu, and Tat Jen Cham. 2010. Face and human gait recognition using image-to-class distance. IEEE Transactions on Circuits \& Systems for Video Technology 20, 3, 431-438.

[97] Toby H. W. Lam, K. H. Cheung, and James N. K. Liu. 2011. Gait flow image: A silhouette-based gait representation for human identification. Pattern Recognition 44, 4, 973-987.

[98] P. Bours and R. Shrestha. 2010. Eigensteps: A giant leap for gait recognition. In International Workshop on Security and Communication Networks. 1-6.

[99] Kjetil Holien. 2008. Gait recognition under non-standard circumstances. Master's Thesis. Gjøvik University, Gjøvik, Norway.

[100] Chiung Ching Ho, C. Eswaran, Kok Why Ng, and June Yee Leow. 2012. An unobtrusive android person verification using accelerometer based gait. In International Conference on Advances in Mobile Computing \& Multimedia. 271-274.

[101] San-Segundo R., Cordoba Ricardo, Ferreiros Javier, and L. F. D’Haro-Enriquez. 2016. Frequency features and GMMUBM approach for gait-based person identification using smartphone inertial signals. Pattern Recognition Letters 73(C), 60-67.

[102] Chunxu Tang and Vir V. Phoha. 2016. An empirical evaluation of activities and classifiers for user identification on smartphones. In IEEE International Conference on Biometrics Theory, Applications and Systems. 1-8.

[103] R. Kumar, V. V. Phoha, and A. Jain. 2015. Treadmill attack on gait-based authentication systems. In IEEE International Conference on Biometrics Theory, Applications and Systems. 1-7.

[104] Paolo Fraccaro, Lorcan Walsh, Julie Doyle, and Dympna O’Sullivan. 2014. Real-world gyroscope-based gait event detection and gait feature extraction. In eTELEMED 2014, The 6th International Conference on eHealth, Telemedicine, and Social Medicine. 247-252.

[105] Changhong Chen, Jimin Liang, Heng Zhao, Haihong Hu, and Jie Tian. 2009. Factorial HMM and parallel HMM for gait recognition. IEEE Transactions on Systems, Man, and Cybernetics, Part C (Applications and Reviews) 39, 1, 114-123.

[106] Wallace Lawson and Zoran Duric. 2009. Analyzing human gait using patterns of translation and rotation. In International Conference Image Analysis and Recognition. 408-417.

[107] Dominik Endres, Enrico Chiovetto, and Martin A. Giese. 2016. Bayesian approaches for learning of primitive-based compact representations of complex human activities. In Dance Notations and Robot Motion. Springer, 117-137.

[108] Qiong Cheng, Bo Fu, and Hui Chen. 2009. Gait recognition based on PCA and LDA. In International Symposium on Computer Science and Computational Technology (ISCSCI'09). Academy Publisher, 124-127.

[109] Su Li Xu and Qian Jin Zhang. 2010. Gait recognition using fuzzy principal component analysis. In International Conference on E-Business and Information System Security. 1-4.

[110] Er Hu Zhang, Hua Bing Ma, Ji Wen Lu, and Ya Jun Chen. 2009. Gait recognition using dynamic gait energy and PCA+LPP method. In International Conference on Machine Learning and Cybernetics. 50-53.

[111] Haiping Lu, K. N. Plataniotis, and A. N. Venetsanopoulos. 2006. Multilinear principal component analysis of tensor objects for recognition. In International Conference on Pattern Recognition. 776-779.

[112] Chiraz BenAbdelkader, Ross Cutler, Harsh Nanda, and Larry Davis. 2001. Eigengait: Motion-based recognition of people using image self-similarity. In Audio-and Video-Based Biometric Person Authentication. Springer, 284-294.

[113] Haiping Lu, K. N. Plataniotis, and A. N. Venetsanopoulos. 2007. Boosting LDA with regularization on MPCA features for gait recognition. In IEEE Biometrics Symposium. 1-6.

[114] Zheyi Fan, Jiao Jiang, Shuqin Weng, Zhonghang He, and Zhiwen Liu. 2016. Human gait recognition based on discrete cosine transform and linear discriminant analysis. In IEEE International Conference on Signal Processing, Communications, and Computing. 1-6.

[115] Ryoichi Kato, Shinji Imakubo, Namiko Akamatsu, Tamotsu Nakatsuchi, Shigeyoshi Nakajima, Hiroyoshi Iwaki, Mitsuhiko Ikebuchi, Yukihide Minoda, and Kunio Takaoka. 2008. Gait analysis by discrete cosine transform (DCT) in patients after total hip arthroplasty using a wearable accelerometer. Fapanese fournal of Clinical Biomechanics 29 , 29, 325-329.

[116] Rubén San-Segundo, Julián David Echeverry-Correa, Cristian Salamea-Palacios, Syaheerah Lebai Lutfi, and José M. Pardo. 2017. I-vector analysis for gait-based person identification using smartphone inertial signals. Pervasive \& Mobile Computing 38, 38, 140-153. 
[117] Dacheng Tao, Xuelong Li, Xindong Wu, and Stephen J. Maybank. 2007. General tensor discriminant analysis and Gabor features for gait recognition. IEEE Transactions on Pattern Analysis \& Machine Intelligence 29, 10, 1700.

[118] M. Pushpa Rani and G. Arumugam. 2010. An efficient gait recognition system for human identification using modified ICA. International fournal of Computer Science \& Information Technology 2, 1, 55-67.

[119] Bo Ye and Yu Mei Wen. 2008. Gait recognition based on DWT and SVM. In International Conference on Wavelet Analysis and Pattern Recognition. 1382-1387.

[120] Stuart D. Mowbray and Mark S. Nixon. 2003. Automatic gait recognition via fourier descriptors of deformable objects. In International Conference on Audio-and Video-Based Biometric Person Authentication. Springer, Berlin, Heidelberg, 566-573.

[121] P. S. Huang, C. J. Harris, and M. S. Nixon. 1999. Human gait recognition in canonical space using temporal templates. IEEE Proceedings-Vision Image and Signal Processing 146, 2, 93-100.

[122] Imed Bouchrika and Mark S. Nixon. 2008. Exploratory factor analysis of gait recognition. In IEEE International Conference on Automatic Face \& Gesture Recognition. 1-6.

[123] Zhihui Lai, Yong Xu, Zhong Jin, and David Zhang. 2014. Human gait recognition via sparse discriminant projection learning. IEEE Transactions on Circuits \& Systems for Video Technology 24, 10, 1651-1662.

[124] Vera Miler Jerković, Milica Djurić-Jovičić, and Mirjana B. Popović. 2013. PCA sensitivity: The role of representative and outlier strides in gait sequence. In Symposium on Neural Network Applications in Electrical Engineering. 123-126.

[125] Eman Mashagba. 2014. Simple and efficient marker-based approach in human gait analysis using Gaussian mixture model. Australian fournal of Basic \& Applied Sciences 8, 1, 137-147.

[126] Miler Jerkovic Vera, Bojanic Dubravka, Jorgovanovic Nikola, Ilic Vojin, and Petrovacki Balj Bojana. 2013. Detecting and removing outlier(s) in electromyographic gait-related patterns. Fournal of Applied Statistics 40, 6, 1319-1332.

[127] Yunfeng Wu and Sridhar Krishnan. 2010. Statistical analysis of gait rhythm in patients with Parkinson's disease. IEEE Transactions on Neural Systems \& Rehabilitation Engineering, A Publication of the IEEE Engineering in Medicine \& Biology Society 18, 2, 150 .

[128] A. Ghebleh and M. Ebrahimi Moghaddam. 2017. Clothing-invariant human gait recognition using an adaptive outlier detection method. Multimedia Tools \& Applications 13, 1-21.

[129] Ikenna Odinaka, Po Hsiang Lai, Alan D. Kaplan, Joseph A. O’Sullivan, Erik J. Sirevaag, Sean D. Kristjansson, Amanda K. Sheffield, and John W. Rohrbaugh. 2011. ECG biometrics: A robust short-time frequency analysis. In IEEE International Workshop on Information Forensics and Security. 1-6.

[130] Claudia Nickel, Tobias Wirtl, and Christoph Busch. 2012. Authentication of smartphone users based on the way they walk using k-NN algorithm. In 8th International Conference on Intelligent Information Hiding and Multimedia Signal Processing. 16-20.

[131] N. V. Boulgouris, K. N. Plataniotis, and D. Hatzinakos. 2005. Gait recognition using dynamic time warping. In 2004 IEEE Workshop on Multimedia Signal Processing. 263-266.

[132] J. Barth, C. Oberndorfer, C. Pasluosta, S. Schĺźlein, H. Gassner, S. Reinfelder, P. Kugler, D. Schuldhaus, J. Winkler, and J. Klucken. 2015. Stride segmentation during free walk movements using multi-dimensional subsequence dynamic time warping on inertial sensor data. Sensors 15, 3, 6419.

[133] Taranjot Kaur Bajwa, Sourav Garg, and Kumar Saurabh. 2017. Gait analysis for identification by using SVM with k-NN and NN techniques. In 4th International Conference on Parallel, Distributed and Grid Computing.

[134] Springer Shmuel and Yogev Seligmann Galit. 2016. Validity of the kinect for gait assessment: A focused review. Sensors 16, 2, 194.

[135] R. Saether, J. L. Helbostad, L. Adde, S. Braendvik, S. Lydersen, and T. Vik. 2015. The relationship between trunk control in sitting and during gait in children and adolescents with cerebral palsy. Developmental Medicine \& Child Neurology 57, 4, 344-350.

[136] Tsolmonbaatar Khurelbaatar, Kyungsoo Kim, Su Kyoung Lee, and Yoon Hyuk Kim. 2015. Consistent accuracy in whole-body joint kinetics during gait using wearable inertial motion sensors and in-shoe pressure sensors. Gait \& Posture 42, 1, 65.

[137] Jiwen Lu and Erhu Zhang. 2007. Gait recognition for human identification based on ICA and fuzzy SVM through multiple views fusion. Pattern Recognition Letters 28, 16, 2401-2411.

[138] J. Wu and J. Wang. 2008. PCA-based SVM for automatic recognition of gait patterns. fournal of Applied Biomechanics 24, 1, 83-7.

[139] Marcin Derlatka and Mikhail Ihnatouski. 2010. Decision tree approach to rules extraction for human gait analysis. In International Conference on Artificial Intelligence and Soft Computing. 597-604.

[140] Qing Guo and Dan Jiang. 2015. Method for walking gait identification in a lower extremity exoskeleton based on Q271248 c4.5 decision tree algorithm. International fournal of Advanced Robotic Systems 12, 4, 1-16.

[141] Hongmin Xue and Zhijing Liu. 2013. A non-linear SVM decision tree method for gait classification via flexible measurement. Journal of Convergence Information Technology 8, 10, 600-608. 
[142] Degui Xiao and Lei Yang. 2008. Gait recognition using Zernike moments and bp neural network. In IEEE International Conference on Networking, Sensing and Control. 418-423.

[143] Han Su and Fenggang Huang. 2006. Gait recognition using principal curves and neural networks. In International Symposium on Neural Networks. 238-243.

[144] Munif Alotaibi and Ausif Mahmood. 2016. Improved gait recognition based on specialized deep convolutional neural networks. In Applied Imagery Pattern Recognition Workshop. 1-7.

[145] Shiqi Yu, Daoliang Tan, and Tieniu Tan. 2006. A framework for evaluating the effect of view angle, clothing and carrying condition on gait recognition. In International Conference on Pattern Recognition. 441-444.

[146] Shiqi Yu, Sen Jia, and Chunyan Xu. 2016. Convolutional neural networks for hyperspectral image classification. Neurocomputing 219, 219, 88-98.

[147] A. Sokolova and A. Konushin. 2017. Gait recognition based on convolutional neural networks. In International Archives of the Photogrammetry, Remote Sensing \& Spatial Information Sciences. 42, 42, 207-212.

[148] Francisco Manuel Castro, Manuel J. Marín-Jiménez, Nicolás Guil, and Nicolás Pérez De La Blanca. 2017. Automatic learning of gait signatures for people identification. In International Work-Conference on Artificial Neural Networks. 257-270.

[149] Weiwei Xing, Ying Li, and Shunli Zhang. 2018. View-invariant gait recognition method by three-dimensional convolutional neural network. fournal of Electronic Imaging 27, 1, 1-11.

[150] Matteo Gadaleta and Michele Rossi. 2018. IDnet: Smartphone-based gait recognition with convolutional neural networks. Pattern Recognition 74, 74, 25-37.

[151] Giacomo Giorgi, Fabio Martinelli, Andrea Saracino, and Mina Sheikhalishahi. 2017. Try walking in my shoes, if you can: Accurate gait recognition through deep learning. In International Conference on Computer Safety, Reliability, and Security. 384-395.

[152] O. Dehzangi, M. Taherisadr, and R. Changalvala. 2017. IMU-based gait recognition using convolutional neural networks and multi-sensor fusion. Sensors 17, 12, 2735.

[153] Y. Zhao and S. Zhou. 2017. Wearable device-based gait recognition using angle embedded gait dynamic images and a convolutional neural network. Sensors 17, 3, 478.

[154] Z. Liu and S. Sarkar. 2006. Improved gait recognition by gait dynamics normalization. IEEE Transactions on Pattern Analysis \& Machine Intelligence 28, 6, 863-876.

[155] Hany Hazfiza Manap, Nooritawati Md Tahir, and R. Abdullah. 2012. Anomalous gait detection using Naive Bayes classifier. In Industrial Electronics and Applications. 378-381.

[156] T. Chalidabhongse, V. Kruger, and R. Chellappa. 2001. The UMD database for human identification at a distance. Technical Report, University of Maryland.

[157] P. Jonathon Phillips, Patrick Grother, Sudeep Sarkar, Isidro Robledo, and Kevin Bowyer. 2002. Baseline results for the challenge problem of human ID using gait analysis. In IEEE International Conference on Automatic Face and Gesture Recognition. 137.

[158] Mark S. Nixon and John N. Carter. 2007. Automatic recognition by gait. Proceedings of the IEEE 94, 11, 2013-2024.

[159] Martin Hofmann, Jürgen Geiger, Sebastian Bachmann, Björn Schuller, and Gerhard Rigoll. 2014. The TUM gait from audio, image and depth (GAID) database: Multimodal recognition of subjects and traits. Fournal of Visual Communication \& Image Representation 25, 1, 195-206.

[160] Pierluigi Casale, Oriol Pujol, and Petia Radeva. 2012. Personalization and user verification in wearable systems using biometric walking patterns. Personal \& Ubiquitous Computing 16, 5, 563-580.

[161] Muhammad Muaaz and Claudia Nickel. 2012. Influence of different walking speeds and surfaces on accelerometerbased biometric gait recognition. In International Conference on Telecommunications and Signal Processing. 508-512.

[162] Thanh Trung Ngo, Yasushi Makihara, Hajime Nagahara, Yasuhiro Mukaigawa, and Yasushi Yagi. 2014. The largest inertial sensor-based gait database and performance evaluation of gait-based personal authentication. Pattern Recognition 47, 1, 228-237.

[163] Jorge L. Reyes-Ortiz, Luca Oneto, Xavier Parra, and Davide Anguita. 2016. Transition-aware human activity recognition using smartphones. Neurocomputing 171(C), 754-767.

[164] Meir Plotnik, Daniel Roggen, Inbal Maidan, Jeffrey M. Hausdorff, and Nir Giladi. 2010. Wearable assistant for Parkinson's disease patients with the freezing of gait symptom. IEEE Transactions on Information Technology in Biomedicine $14,2,436-446$.

[165] Abdenour Hadid, Mohammad Ghahramani, Vili Kellokumpu, Xiaoyi Feng, John Bustard, and Mark Nixon. 2015. Gait biometrics under spoofing attacks: An experimental investigation. Journal of Electronic Imaging 24, 6, 063022.

[166] A. Hadid, M. Ghahramani, J. Bustard, and M. Nixon. 2012. Improving gait biometrics under spoofing attacks. 21st International Conference on Pattern Recognition (ICPR 2012). 1-10.

[167] A. Hadid, M. Ghahramani, V. Kellokumpu, M. Pietikainen, J. Bustard, and M. Nixon. 2012. Can gait biometrics be spoofed? 21st International Conference on Pattern Recognition (ICPR 2012). 3280-3283. 
[168] Bendik B. Mjaaland, Patrick Bours, and Danilo Gligoroski. 2010. Walk the walk: attacking gait biometrics by imitation. In International Conference on Information Security. 361-380.

[169] Muhammad Muaaz and Rene Mayrhofer. 2017. Smartphone-based gait recognition: From authentication to imitation. IEEE Transactions on Mobile Computing 16, 11, 3209-3221.

[170] Davrondzhon Gafurov. 2007. Security analysis of impostor attempts with respect to gender in gait biometrics. In IEEE International Conference on Biometrics: Theory, Applications, and Systems. 1-6.

[171] Galina V. Veres, Mark S. Nixon, and John N. Carter. 2005. Modelling the time-variant covariates for gait recognition. In International Conference on Audio- and Video-Based Biometric Person Authentication. 597-606.

[172] Zongyi Liu and Sudeep Sarkar. 2004. Simplest representation yet for gait recognition: Averaged silhouette. In International Conference on Pattern Recognition, Vol. 4. 211-214.

[173] Z. Liu and S. Sarkar. 2005. Effect of silhouette quality on hard problems in gait recognition. IEEE Transactions on Systems. Man, and Cybernetics, Part B (Cybernetics). A Publication of the IEEE Systems, Man, and Cybernetics Society $35,2,170-183$

[174] Zongyi Liu, Laura Malave, and Sudeep Sarkar. 2004. Studies on silhouette quality and gait recognition. In IEEE Computer Society Conference on Computer Vision and Pattern Recognition. 704-711.

[175] Darko S. Matovski, Mark S. Nixon, Sasan Mahmoodi, and John N. Carter. 2012. The effect of time on gait recognition performance. IEEE Transactions on Information Forensics \& Security 7, 2, 543-552.

[176] K. Bashir, Tao Xiang, and Shaogang Gong. 2010. Gait recognition using gait entropy image. In International Conference on Crime Detection and Prevention. 1-6.

[177] Galina V. Veres, Mark S. Nixon, and John N. Carter. 2005. Model-based approaches for predicting gait changes over time. In International Conference on Advances in Biometric Person Authentication. 213-220.

[178] S. Samangooei, J. Bustard, M. S. Nixon, and J. N. Carter. 2011. On acquisition and analysis of a dataset comprising of gait, ear and semantic data. Multibiometrics for Human Identification. Cambridge University Press, 277-301.

[179] Yu Guan, Chang Tsun Li, and Yongjian Hu. 2012. Robust clothing-invariant gait recognition. In 8th International Conference on Intelligent Information Hiding and Multimedia Signal Processing. 321-324.

[180] Jian Yang, David Zhang, Alejandro F. Frangi, and Jingyu Yang. 2004. Two-dimensional PCA: A new approach to appearance-based face representation and recognition. IEEE Transactions on Pattern Analysis \& Machine Intelligence $26,1,131-137$

[181] Md. Altab Hossain, Yasushi Makihara, Junqiu Wang, and Yasushi Yagi. 2010. Clothing-invariant gait identification using part-based clothing categorization and adaptive weight control. Pattern Recognition 43, 6, 2281-2291.

[182] Muhammad Rasyid Aqmar, Koichi Shinoda, and Sadaoki Furui. 2010. Robust gait recognition against speed variation. In 20th IEEE International Conference on Pattern Recognition (ICPR'10). 2190-2193.

[183] Takumi Kobayashi and Nobuyuki Otsu. 2009. Three-way auto-correlation approach to motion recognition. Pattern Recognition Letters 30, 3, 212-221.

[184] Seungkyu Lee, Yanxi Liu, and Robert Collins. 2007. Shape variation-based frieze pattern for robust gait recognition In IEEE Conference on Computer Vision and Pattern Recognition (CVPR'07). 1-8.

[185] J. D. Shutler, M. G. Grant, M. S. Nixon, and J. N. Carter. 2004. On a large sequence-based human gait database. In Applications and Science in Soft Computing. Springer, Berlin, Heidelberg, 339-346.

[186] I. Robledo, P. Grother, P. J. Phillips, S. Sarkar, and K. W. Bowyer. 2002. The gait identication challenge problem: data sets and baseline algorithm. In Proceedings of the 16th International Conference on Pattern Recognition (ICPR'02) 385-388.

[187] Yu Guan, Chang Tsun Li, and S. D. Choudhury. 2013. Robust gait recognition from extremely low frame-rate videos. In International Workshop on Biometrics and Forensics. 1-4.

[188] Atsushi Mori, Yasushi Makihara, and Yasushi Yagi. 2010. Gait recognition using period-based phase synchronization for low frame-rate videos. In International Conference on Pattern Recognition. 2194-2197.

[189] Yasushi Makihara, Atsushi Mori, and Yasushi Yagi. 2010. Temporal super resolution from a single quasi-periodic image sequence based on phase registration. In Asian Conference on Computer Vision. 107-120.

[190] Yasushi Makihara, Hidetoshi Mannami, and Akira Tsuji. 2012. The OU-ISIR gait database comprising the treadmill dataset. IPSf Transactions on Computer Vision \& Applications 4, 53-62.

[191] John N. Carter and Mark S. Nixon. 2000. On measuring trajectory-invariant gait signatures. In Proceedings of 19th Congress of the International Society for Photogrammetry and Remote Sensing. 114-121.

[192] Nicholas M. Spencer and John N. Carter. 2002. Viewpoint invarience in automatic gait recognition. Proc. AutoID 1-6.

[193] Yasushi Makihara, Ryusuke Sagawa, Yasuhiro Mukaigawa, Tomio Echigo, and Yasushi Yagi. 2006. Gait recognition using a view transformation model in the frequency domain. European Conference on Computer Vision. Springer Berlin, 151-163.

[194] Shiqi Yu, Tieniu Tan, Kaiqi Huang, Kui Jia, and Xinyu Wu. 2009. A study on gait-based gender classification. IEEE Transactions on Image Processing 18, 8, 1905-1910. 
[195] Zhaojun Xue, Dong Ming, Wei Song, Baikun Wan, and Shijiu Jin. 2010. Infrared gait recognition based on wavelet transform and support vector machine. Pattern Recognition 43, 8, 2904-2910.

[196] Jiwen Lu and Yap Peng Tan. 2010. Uncorrelated discriminant simplex analysis for view-invariant gait signal computing. Pattern Recognition Letters 31, 5, 382-393.

[197] Xin Geng, Liang Wang, Ming Li, Qiang Wu, and Kate Smithmiles. 2008. Adaptive fusion of gait and face for human identification in video. In IEEE Workshop on Applications of Computer Vision (WACV'08). 1-6.

[198] Davrondzhon Gafurov, Einar Snekkenes, and Patrick Bours. 2007. Spoof attacks on gait authentication system. IEEE Transactions on Information Forensics \& Security 2, 3, 491-502.

Received January 2018; revised May 2018; accepted May 2018 